\title{
1 Auto-regulatory J-domain interactions control Hsp70 recruitment to 2 the DnaJB8 chaperone
}

4 Bryan D. Ryder ${ }^{1,2}$, Irina Matlahov ${ }^{3,5}$, Sofia Bali ${ }^{1,2}$, Jaime Vaquer-Alicea ${ }^{2,4}$, Patrick C.A. van der 5 Wel $^{3,5,{ }^{*},}$, Lukasz A. Joachimiak ${ }^{2,6,{ }^{*}}$

6

${ }^{1}$ Molecular Biophysics Graduate Program, University of Texas Southwestern Medical Center, Dallas, TX 75390,

8 United States

92 Center for Alzheimer's and Neurodegenerative Diseases, University of Texas Southwestern Medical Center, Dallas,

10 TX 75390, United States

$11{ }^{3}$ Department of Structural Biology, University of Pittsburgh School of Medicine, Pittsburgh, PA 15213, USA

$12{ }^{4}$ Neuroscience Graduate Program, University of Texas Southwestern Medical Center, Dallas, TX 75390, United

13 States

$14{ }^{5}$ Zernike Institute for Advanced Materials, University of Groningen, 9747 AG Groningen, Netherlands

$15{ }^{6}$ Department of Biochemistry, University of Texas Southwestern Medical Center, Dallas, TX 75390, United States

16 * Correspondence: Lukasz.Joachimiak@utsouthwestern.edu, p.c.a.van.der.wel@rug.nl 


\section{Abstract}

34 The Hsp40/Hsp70 chaperone families combine versatile folding capacity with high

35 substrate specificity, which is mainly facilitated by Hsp40s. The structure and function of

36 many Hsp40s remain poorly understood, particularly oligomeric Hsp40s that suppress

37 protein aggregation. Here, we used a combination of biochemical and structural

38 approaches to shed new light on the domain interactions of the Hsp40 DnaJB8, and how

39 they regulate recruitment of partner Hsp70s. We identify an interaction between the J-

40 Domain (JD) and C-terminal domain (CTD) of DnaJB8 that sequesters the JD surface,

41 preventing Hsp70 interaction. We propose a new model for DnaJB8-Hsp70 regulation,

42 whereby the JD-CTD interaction of DnaJB8 acts as a reversible autoinhibitory switch that

43 can control the binding of Hsp70. These findings suggest that the evolutionarily conserved

44 CTD of DnaJB8 is a regulatory element of chaperone activity in the proteostasis network. 


\section{INTRODUCTION}

61 The cellular chaperone network needs to handle a diversity of protein substrates in

62 numerous different (mis)folded states. This demands a combination of broad versatility

63 and specificity in terms of substrate recognition, even though the central players Hsp70

64 and Hsp90 are highly conserved. This apparent contradiction is resolved by the Hsp40 65 (DnaJ) family of proteins, which are chaperones that recruit and regulate the activity of 66 Hsp70 chaperones in refolding misfolded proteins ${ }^{1-4}$. While the human Hsp70 family is 67 highly conserved, the Hsp40 chaperone family encodes 47 diverse members, each with 68 specialized functions in substrate recognition and presumed coordination with Hsp705-7.

69 DnaJ proteins feature a J-domain (JD), which binds to Hsp70s through a conserved 70 electrostatic interaction to trigger ATP hydrolysis by the Hsp707-10. This initiates a 71 conformational rearrangement in the Hsp70 substrate binding domain that helps capture 72 the substrate for folding, refolding, or disaggregation ${ }^{11}$. When misfolded proteins cannot 73 be refolded, some Hsp40s help direct them for degradation ${ }^{12-13}$.

75 In humans, Hsp40s function as monomers, dimers, or oligomers. Classical Hsp40 76 members assemble into homo-dimers or hetero-dimers ${ }^{14}$ through conserved C-terminal 77 motifs and bind unfolded substrates through conserved $\beta$-barrel $\mathrm{C}$-terminal domains 78 (CTD) ${ }^{15}$. A subset of non-classical Hsp40s, including DnaJB2, DnaJB6b, DnaJB7 and 79 DnaJB8 have a domain architecture that is distinct from the classical dimeric DnaJ 80 orthologs ${ }^{14-17}$. These Hsp40s retain the JD, but have distinct other domains including 81 substantial differences in their CTD structures. Of these, the DnaJB8 and DnaJB6b 82 proteins self-assemble in vitro and in vivo ${ }^{16,18-19}$. The role of their CTD remains unclear, 83 as the literature suggests that it either drives oligomerization or mediates intramolecular 84 contacts $^{16-18,20}$. The oligomers' structural and dynamic heterogeneity has greatly hindered 85 efforts to study them, yielding for DnaJB6 limited-resolution cryoEM data ${ }^{19}$ or requiring 86 invasive deletion mutations to gain structural insight in soluble mutant variants ${ }^{16-17}$. 
88 Here we examine DnaJB8, which has been shown to be particularly effective at

89 preventing polyglutamine (polyQ) deposition, even more so than the homologous

90 DnaJB6b, despite $63 \%$ sequence identity $16,18,20-21$. This indicates that their specific

91 modes of activity are distinct in spite of their similarities in sequence and domain

92 arrangement. Notably, unlike other chaperones that inhibit mutant huntingtin

93 aggregation ${ }^{22}$, DnaJB8 and DnaJB6b are thought to bind directly to polyQ elements and

94 thus are active across the whole family of polyQ diseases ${ }^{16,21}$. The two proteins have

95 different expression profiles, with DnaJB8 being highly expressed in testes, while

96 DnaJB6b is ubiquitous, which in part explains the deeper knowledge available for the

97 latter protein. While both DnaJB6b and DnaJB8 assemble into soluble oligomers ${ }^{16,18-19,}$

9821 , DnaJB8 in particular displays a higher propensity to assemble ${ }^{16}$. Here, we applied a

99 multidisciplinary approach to understand the architecture and dynamics of DnaJB8 in

100 cells and in vitro. We used crosslinking mass spectrometry (XL-MS) to identify local intra-

101 domain contacts and long-range contacts. Guided by modeling, we mutated aromatic

102 residues to create a monomeric mutant that maintains the intramolecular domain contacts

103 observed in oligomers in cells and in vitro. Solid-state NMR (ssNMR) probed the structural

104 and dynamic order of the solvated oligomers, to reveal dramatic domain-specific

105 differences in (dis)order and a lack of highly flexible regions. Electrostatic interactions

106 control the JD transitioning between an ordered immobilized state and a more mobilized

107 state, which we attribute to JD - CTD interactions that we reconstitute with isolated

108 domains and detect in full-length protein. Finally, we demonstrate that the JD-CTD

109 contacts regulate the recruitment of $\mathrm{Hsp} 70$, representing a built-in regulatory mechanism

110 that controls recruitment (and thus activation) of Hsp70.

\section{RESULTS}

\section{DnaJB8 domain interactions in a cellular context}

114 DnaJB8 encodes three domains C-terminal to the JD (Fig. 1a): a glycine and 115 phenylalanine (G/F) rich domain (Fig. 1a, blue), a serine/threonine rich (S/T) domain (Fig. 116 1a, cyan) and a C-terminal CTD (Fig. 1a, green). Prior studies have highlighted the ability 
117 of DnaJB8 to assemble into oligomers but little is known about DnaJB8 domain

118 interactions in cells ${ }^{16,20}$. We expressed DnaJB8 fused to a green fluorescent protein

119 (GFP) derivative mClover3 (herein, DnaJB8-Cover) in HEK293 cells (Fig. 1a). DnaJB8-

120 Clover expression leads to the formation of fluorescent juxtanuclear puncta with an

121 approximate maximum diameter of $1.0 \mu \mathrm{m}$ (Fig. 1b) in $39.2 \pm 3.1 \%$ of the cells (Fig. 1c)

122 while Clover alone expression yielded diffuse fluorescence (Fig. 1b) with little to no puncta

123 (Fig. 1c; $0.44 \pm 0.50 \%$ ). The puncta observed in these cells indicate the presence of

124 ordered aggregates, while the more uniformly dispersed signal is indicative of soluble

125 oligomers and monomers. Decreasing the DnaJB8-Clover expression 3-fold as

126 determined by western blot (Supplementary Fig. 1a) and fluorescence intensity

127 (Supplementary Fig. 1b) yielded only a 2-fold decrease in the number of puncta

128 (16.2 $\pm 0.08 \%$; Fig. 1c). The frequency of puncta for Clover alone remained below $1 \%$ in

129 both experiments (Fig. 1C). Thus, even at reduced levels of expression DnaJB8 can form

130 puncta in cells.

132 We next sought to characterize biochemical properties of DnaJB8-Clover expressed in 133 mammalian cells. DnaJB8-Clover protein was purified using $\alpha$-GFP nano-bodies ${ }^{23-24}$

134 (Supplementary Fig. 1c). To gain insight into the topology of DnaJB8, we employed a XL135 MS approach to define contacts between different domains ${ }^{25-27}$. Isolated DnaJB8-Clover 136 was reacted with adipic acid dihydrazide (ADH) and 4-(4,6-dimethoxy-1,3,5-triazin-2-yl)137 4-methyl-morpholinium chloride (DMTMM). ADH covalently links carboxylate-carboxylate 138 contacts via a 6-carbon bridge, while DMTMM forms a direct covalent bond between 139 lysine-carboxylate groups through dehydration ${ }^{26}$. Crosslinking treatment of the purified 140 soluble DnaJB8-Clover species revealed predominantly monomers and dimers in the 141 cells, with a trace of larger oligomers (Supplementary Fig. 1d). We identified 21 crosslinks 142 that parsed into 3 regions: JD-JD, CTD-CTD and JD-CTD (Fig. 1d, Supplementary Data 143 1). The three local JD contacts (Fig. 1d; red box) are consistent with its experimental 144 structure (Supplementary Fig. 1e). Local CTD contacts (Fig. 1d, green box) were also 145 accompanied by inter-domain JD-CTD contacts that localize to helices 2 and 3 of JD (Fig. $1461 \mathrm{~d}$, grey box). Additionally, we identified a contact between the JD and a putative helix 5 
147 (Fig. 1d, H5) of the G/F domain, as also recently identified in DnaJB6b ${ }^{17}$. Thus, soluble

148 DnaJB8-Clover species isolated from mammalian cells reveal an array of inter-domain

149 interactions, including contacts between the charge complementary JD and CTD.

\section{DnaJB8 domain contacts are preserved in vitro}

152 For a more detailed understanding of DnaJB8 domain architecture in vitro, we produced 153 recombinant DnaJB8 as described previously ${ }^{16}$. We first used Dynamic Light Scattering 154 (DLS) to monitor the hydrodynamic radius $\left(R_{h}\right)$ of DnaJB8 species over time. The 155 scattering data reveals bona fide DnaJB8 sizes which begin as a small $4.28 \pm 0.82 \mathrm{~nm}$ 156 species with a small (<\%1 by mass) contribution of larger species ( $>10 \mathrm{~nm})$ but over time 157 these small species shift to $5.35 \pm 0.22 \mathrm{~nm}$ at 10 hours and to $5.77 \pm 0.43 \mathrm{~nm}$ after 20 hours 158 (Fig. 1e and Supplementary Fig. 1f). Over the time course, a fraction of the soluble small 159 species converted into larger oligomers $>10 \mathrm{~nm}$ (30.6\% by mass) with an average $\mathrm{Rh}$ of $16090 \mathrm{~nm}$ (Fig. 1e and Supplementary Fig. 1f). These findings are consistent with prior studies 161 on DnaJB6b and DnaJB8 showing that they have the capacity to assemble into 162 polydisperse soluble oligomers in vitro ${ }^{16,18-19,21,28 .}$

164 Next, we aimed to better understand the topology of DnaJB8 in vitro using XL-MS, 165 employing two parallel chemistries; disuccinimidyl suberate (DSS) and ADH/DMTMM on 166 samples after a brief 30 mins of incubation. Consistent with the DLS data at early time 167 points, we observe by SDS-PAGE a ladder of bands indicating the formation of covalent 168 intermolecular contacts dominated by a dimer (Supplementary Fig. 1g). XL-MS analysis 169 of these samples showed only 3 crosslinks in the DSS condition (Fig. 1f, Supplementary 170 Data 1). In contrast, the ADH/DMTMM analysis yielded 24 crosslinks (Fig. 1f, 171 Supplementary Data 1). Importantly, this XL-MS pattern persisted across the DLS time 172 course (Supplementary Fig. 1h and Supplementary Data 1) and closely matches the pairs 173 observed in the assemblies recovered from the mammalian cells including crosslinks from 174 both JD and CTD to H5 (Fig. 1d, H5). The JD crosslinks are consistent with the structure 175 of the domain (Supplementary Fig. 1i) ${ }^{26}$. 
177 The CTD yielded 8 crosslinks (Fig. 1f). Amongst these, the locally-linked regions spanning 178 E208-E211 and K223-K227 are central to the CTD and repeatedly react to peripheral 179 sites. The third cluster of contacts linked the distal JD and CTD through 4 crosslinks (Fig.

180 1f; JD:CTD). The interdomain CTD crosslink sites are exclusively mediated through acidic 181 amino acids; D212, E209 and E211. Interestingly, the crosslinked amino acids on the JD 182 are all lysines that localize to basic surfaces along helix 1 and 2 (Supplementary Fig. 1j) 183 and overlap the basic Hsp70 binding surface ${ }^{10}$. These XL-MS data identify an intricate 184 network of electrostatic inter-domain interactions in both monomeric and oligomeric 185 DnaJB8.

187 To further test the apparent role of electrostatically driven interactions, we used a higher 188 ionic strength buffer in an analogous series of experiments. Using DLS we observed a 189 defined species with a $7.75 \pm 0.7 \mathrm{~nm}$ size in $285 \mathrm{mM} \mathrm{NaCl}$ (Fig. 1g, Supplementary Data 2), 190 which are more expanded compared to species in 150mM NaCl (Fig. 1e, Supplementary 191 Data 2). XL-MS analysis recapitulates the short-range contacts within the JD and CTD 192 domains but the JD:CTD contacts were notably absent (Fig. 1h). To control for reactivity 193 in each condition we compared the frequency of ADH-driven singly reacted modifications, 194 called monolinks. These data show nearly identical numbers of modifications suggesting 195 that the reactivity between these two conditions is nearly identical (Supplementary Fig. $1961 \mathrm{k})$. Thus, the disruption of electrostatically driven interactions is accompanied by 197 changes in the domain architecture.

199 JD:CTD interaction is mediated by electrostatic contacts

200 To understand how the JD and CTD domains could be interacting, we used Rosetta 201 modeling guided by XL-MS restraints. We built a starting model by combining the 202 experimental structure of the JD (PDBID: 2DMX) with an ab initio-derived model for CTD 203 and the middle domains fully extended. The starting model was then collapsed by 204 applying the JD-CTD crosslinks as restraints (Fig. 2a and Supplementary Fig. 2a-b). The 205 fully expanded monomer collapsed from a predicted $R_{h}$ of $9.27 \mathrm{~nm}\left(\mathrm{R}_{\mathrm{g}}, 6.65 \mathrm{~nm}\right)$ to 4.02 
$206 \mathrm{~nm}\left(\mathrm{R}_{\mathrm{g}}, 2.45 \mathrm{~nm}\right)$ (Fig. 2a). Comparing these values to our DLS radii in $150 \mathrm{mM} \mathrm{NaCl}$ 207 suggests that the dominant species are likely monomers and dimers. The DLS 208 measurements in $285 \mathrm{mM} \mathrm{NaCl}$ are consistent with the initial expanded model with the 209 JD:CTD contacts disengaged. Thus, our data support that DnaJB8 exists in solution as 210 small soluble species (4 to $6 \mathrm{~nm}$ ), dominated by monomer/dimer but with capacity to form 211 larger oligomers over time, both in vitro and in vivo.

213 Guided by the constraints, the final model "docks" the JD onto the CTD placing a putative 214 acidic surface on the CTD in contact with the basic surface on the JD (Fig. $2 a$ and 215 Supplementary Fig. 2b) and additionally bringing $\mathrm{H} 5$ in proximity to both the JD and CTD 216 as similarly observed for DnaJB6b (Supplementary Fig. 2c) ${ }^{17}$. The CTD has proximal 217 basic surfaces that flank its acidic surface, generating a characteristic alternating charge 218 pattern that is inverted on the JD (Fig. 2c). Mapping sequence conservation onto the 219 Rosetta-generated model, we find that these JD:CTD contacts are largely conserved 220 (Supplementary Fig. 2d). In a co-evolution analysis using the Gremlin algorithm ${ }^{29-31}$ we $^{2}$ 221 identified amino acid positions that covary. Not only did we observe many amino acid 222 pairs that covary between the JD and CTD as well as H5, but our XL-MS pairs overlap 223 with these covarying positions (Fig. 2d). The similarity between the predicted covarying 224 contacts, conservation, and the XL-MS experimental contacts strengthens our DnaJB8 225 JD-CTD model, and suggests that XL-MS can detect functionally important interaction 226 sites.

\section{JD:CTD contacts are present in monomeric DnaJB8}

229 In our "collapsed" monomer structural model the 17 phenylalanine residues in the G/F 230 and $\mathrm{S} / \mathrm{T}$ domains were predicted to be in part solvent exposed (Fig. 2a, spheres). We 231 hypothesized that these aromatic residues may play a role in DnaJB8 assembly and 232 engineered a mutant, in which all $\mathrm{G} / \mathrm{F}$ - and S/T-region phenylalanine residues were 233 mutated to serine residues (Fig. 2e, herein DnaJB8 $\mathrm{F}_{\rightarrow}$ ). Using our DLS and XL-MS 234 pipeline, we evaluated assembly of DnaJB8 $\mathrm{F}_{\rightarrow} \mathrm{s}$. By DLS, the DnaJB8 $\mathrm{F}_{\rightarrow} \mathrm{S}$ mutant 
235 remained stable as a $3.53 \pm 0.05 \mathrm{~nm}$ species over 21 hours (Fig. 2f, Supplementary Data 236 2). SDS-PAGE of crosslinked DnaJB8 $\mathrm{F}_{\rightarrow} \mathrm{S}$ also showed no intermolecular crosslinks. 237 (Supplementary Fig. 2e). Size Exclusion Chromatography Multi-Angle Light Scattering 238 (SEC-MALS) analysis on DnaJB8 $\mathrm{F}_{\rightarrow}$ S revealed it to be a monomer with a molecular weight 239 of $24,530 \pm 30 \mathrm{~g} / \mathrm{mol}$ (Fig. $2 \mathrm{~g}$ ). These data support that phenylalanine residues in the $\mathrm{G} / \mathrm{F}$ 240 and S/T domains play a role in higher order assembly. Next, we used XL-MS to test 241 whether this DnaJB8 $\mathrm{F}_{\rightarrow} \mathrm{s}$ monomer maintained the intramolecular JD and CTD contacts 242 observed in WT DnaJB8 (Fig. 2h). Analysis of the crosslinked DnaJB8 $\mathrm{F}_{\rightarrow}$ s, revealed 243 identical local crosslinks within JD and CTD and also detected 3 crosslinks between the 244 JD and CTD. Interestingly, in DnaJB8 $\mathrm{F}_{\rightarrow}$ s, the H5 crosslinks to JD were absent, consistent 245 with requirement of a phenylalanine in $\mathrm{H} 5$ for binding to the JD (Fig. 2h, H5). The 246 presence of the JD-CTD crosslinks in a monomeric mutant marks them to represent 247 intramolecular JD:CTD interactions.

We can now use the experimental DLS radii with our structural models to more accurately 250 infer the dimensions of the small soluble DnaJB8 species (Fig. 2i). At the start of the WT 251 DnaJB8 DLS time course, we observed an initial population of polydisperse particles with 252 an average radius of $4.28 \pm 0.82 \mathrm{~nm}$ (Fig. 1e). The DnaJB8 $\mathrm{F} \rightarrow \mathrm{S}$ mutant showed a radius of $2533.53 \pm 0.05 \mathrm{~nm}$ with a very narrow monodisperse distribution, further supporting our model 254 of a monomer "collapsed" by JD:CTD interactions. Based on a model proposed by Marsh 255 and Forman-Kay ${ }^{32}$, we also estimate that a monomeric 232-residue DnaJB8 protein 256 should have a size of $3.98 \mathrm{~nm}$. These data support our analysis that WT DnaJB8 at first 257 adopts primarily a monomer/dimer distribution that has the capacity to then assemble into 258 large oligomers. In contrast, the larger DLS $R_{h}$ values measured for DnaJB8 in 285mM $259 \mathrm{NaCl}$ (Fig. 1g), are a result of the loss of the JD:CTD contacts yielding a small oligomer 260 mediated by aromatic contacts.

\section{2 ssNMR on DnaJB8 oligomers reveals regions of disorder and order}

263 For additional insight into their molecular structure and dynamics, magic-angle-spinning 
264 (MAS) ssNMR was performed on the hydrated oligomers of U- ${ }^{13} \mathrm{C},{ }^{15} \mathrm{~N}$-labeled DnaJB8. MAS ssNMR of hydrated protein assemblies allows for the site- and domain-specific detection of mobility and (secondary) structure, even in presence of disorder and heterogeneity. 1D and 2D ssNMR spectra of the DnaJB8 oligomers feature many broad peaks, with linewidths up to $0.38 \mathrm{kHz}$, consistent with an oligomeric assembly displaying structural disorder (Fig. 3a; Supplementary Fig. 3a-d). However, strikingly, distinct subsets of narrow peaks are also detected, with linewidths of 0.1 to $0.2 \mathrm{kHz}$ (Fig. 3c-d left). These ssNMR experiments employ the cross-polarization (CP) technique, in which observable residues must be rigid or immobilized ${ }^{33}$. In INEPT-based ssNMR, which is selective for highly dynamic segments, the oligomers show little signal ${ }^{33-36}$ (more below).

274 Then, the observed narrow signals in CP spectra must originate from an immobilized, 275 well-ordered subset of DnaJB8 residues. These narrow signals are from amino acid 276 types $^{37}$ in the JD while the broad peaks are dominated by signals from residues common 277 in other domains (Supplementary Table 1). The former also reflect mostly $\alpha$-helical 278 structure, while the latter are mostly random coil and $\beta$-sheet ${ }^{38}$. With known chemical 279 shifts of the DnaJB8 JD in solution, we prepared a synthetic 2D spectrum (Supplementary 280 Fig. $3 \mathrm{~b}$ red) that has a striking correspondence to the narrow ssNMR peaks 281 (Supplementary Fig. 3b black), such that we tentatively assign those to residues in helix 2822 and helix 3 . The $2 \mathrm{D}{ }^{15} \mathrm{~N}-{ }^{13} \mathrm{C} \alpha$ (NCA) ssNMR spectrum showed a similar alignment 283 between narrow peaks and JD signals in solution (Supplementary Fig. 3d). These CP284 based 2D spectra also feature strong peaks from immobilized charged side chains (Lys, 285 Arg, Asp, Glu; Supplementary Figure 3e-f), which is consistent with their involvement in 286 salt-bridge interactions predicted by the XL-MS analysis above.

287 In absence of experimental solution NMR data for other domains, we predicted 288 estimated spectra based on our structural models (Fig. 3c-d green; Supplementary Fig. $2893 \mathrm{c})^{39}$. These peak patterns qualitatively resemble the broad signals in our 2D ssNMR 290 data. A particular strength of MAS ssNMR of hydrated proteins is the ability to gauge local 291 and global dynamics. Single pulse excitation (SPE) and refocused INEPT spectra, which 292 enhance the more dynamic parts of samples ${ }^{35-36}$ show surprisingly little evidence of 
293 flexible residues (Fig. 3e top red). Indeed, the main INEPT signal ( $\sim 2 \mathrm{ppm}$ ) is just from 294 solvent-exposed Lys side chains and lacks evidence of flexible protein regions (even from 295 the S/T or G/F regions). Given that the 1D CP and SPE spectra (Fig. 3e top) look similar, 296 with higher signal intensities in the former, the different domains of the protein actually 297 must have a similar degree of mobility and all be mostly immobilized, without flexible 298 regions. Combined, the ssNMR data reveal oligomers that are heterogeneous in structure 299 but lack extended flexible domains. In other words, the central G/F and ST domains are 300 heterogenous, but also immobilized within the oligomers, consistent with the 301 abovementioned role of their Phe residues in driving oligomer assembly. Uniquely 302 ordered are parts of the JD (residues in helices 2 and 3; Supplementary Fig. 3g,h), which 303 show up as well-folded and immobilized.

Interaction sites from ssNMR

306 MAS ssNMR studies of DnaJB8 oligomers in PBS buffer with $285 \mathrm{mM} \mathrm{NaCl}$ (analogous 307 to the studies above) are shown in Fig. 3b. The 2D spectrum reproduces the broad signals 308 of the immobilized oligomers, but the narrow JD peaks are now strikingly absent. 309 Comparing CP and SPE ssNMR spectra (Fig. 3e bottom), there is an increase in overall 310 mobility. Notably, no new "flexible" ssNMR signals were identified by INEPT ssNMR. We 311 attribute the loss of JD signals in CP-based spectra to increased mobility due to disruption 312 of long-range electrostatic interactions, while the lack of INEPT peaks tells us the JD is 313 still folded and partly immobilized by covalent attachment to the overall assembly. In other 314 words, the JD is invisible due to intermediate timescale dynamics ${ }^{33}$, 40 . Since the broad 315 signals from the other domains are preserved, it appears that the core architecture of the 316 oligomers persists, consistent with aromatic and hydrophobic interactions.

\section{Isolated JD and CTD are folded and monomeric}

319 To further characterize the JD and CTD interaction, we produced isolated JD (herein JD ${ }_{1}$ 320 77) and CTD (herein CTD 170-232) (Fig. 4a). SEC analysis of JD ${ }_{1-77}$ and CTD $170-232$ revealed 321 monodispersed peaks (Fig. 4b). SEC-MALS determined each domain to be monomeric 
322 with a molecular weight of $10,220 \pm 220 \mathrm{~g} / \mathrm{mol}$ and $8,376 \pm 14 \mathrm{~g} / \mathrm{mol}$ for $\mathrm{JD}_{1-77}$ and CTD 170 323 232, respectively (Supplementary Fig. 4a-b). Also, by DLS we measured the JD $1-77 R_{h}$ to 324 be $2.31 \pm 0.13 \mathrm{~nm}$ and the $\mathrm{CTD}_{170-232}$ to be $1.71 \pm 0.02 \mathrm{~nm}$, with both stable over 15 hours 325 (Supplementary Fig. 4c, Supplementary Data 2). We again employed XL-MS to probe the 326 individual domains and compare them to full-length protein. On an SDS-PAGE gel, the 327 crosslinked JD1-77 and CTD 170-232 remained monomeric following crosslinking (Fig. 4c). 328 XL-MS analysis yielded 4 crosslinks for JD1-77 and 6 crosslinks for CTD $170-232$ (Fig. 4d, 329 Supplementary Data 1). The identified crosslinks revealed good agreement between the 330 local domain crosslinks observed in the full-length DnaJB8 and the isolated domains (Fig. $3314 d$, Supplementary Fig. 4d).

332 We built an ensemble of models for the CTD $170-232$ using ab initio ROSETTA ${ }^{40}$. The 333 calculated $R_{h}$ for the structural ensemble was consistent with the DLS measurement of $3341.7 \mathrm{~nm}$ (Supplementary Fig. 4g). The models formed a low contact order 5-stranded $\beta$ 335 sheet topology and the $R_{h}$ variation can be attributed to the more flexible termini 336 (Supplementary Fig. 4g, inset). Circular dichroism (CD) on the CTD sample yields spectra 337 consistent with a predominantly $\beta$-sheet content, as predicted by our model 338 (Supplementary Fig. 4e-f). We mapped the 14 CTD-derived crosslinks from across 339 experiments onto the monomeric ensemble, finding that a majority of structures explain $34010-11$ crosslinks but only a single model explains 13 of 14 (Fig. 4e). These crosslink pairs 341 map onto each face of the $\beta$-sheet and the distances are compatible with the geometry 342 of the crosslinking chemistry. The crosslinks that fall outside of the distance cutoff localize 343 to the more dynamic C-terminus of CTD (Fig. 4e and Supplementary Fig. 4g, inset) at 344 positions K227 and K223. The CTD topology is defined by four $\beta$-turns stabilized by 345 conserved asparagine/aspartate-glycine sequences (N/DG) and overlays well with the 346 DnaJB6b CTD (Fig. 4f) ${ }^{17}$. Thus, our data support that both the JD1-77 and CTD 170-232 347 domains are folded, monomeric, and do not have intrinsic assembly properties. 
350 In our experiments on the full-length DnaJB8 oligomers we observed that the JD and CTD 351 interact through complementary electrostatic surfaces. We further probed this interaction 352 by mixing the individual JD $_{1-77}$ and CTD $_{170-232}$ domains in vitro (Fig. 5a). We incubated 353 Flourescein(FITC)-labelled JD $_{1-77}$ with a series of CTD $170-232$ concentrations and 354 measured binding affinity using a fluorescence polarization (FP) assay. The resulting 355 binding curve revealed that the $\mathrm{JD}_{1-77}$ binds to the $\mathrm{CTD}_{170-232}$ with $0.542 \pm 0.071 \mu \mathrm{M}$ affinity 356 (Fig. 5a; bottom). Parallel experiments using Microscale Thermophoresis revealed a $\mathrm{K}_{d}$ 357 of $5.36 \pm 1.15 \mu \mathrm{M}$ (Supplementary Fig. 5a) suggesting that the affinity is in the low 358 micromolar range. $\mathrm{JD}_{1-77}$ and $\mathrm{CTD}_{170-232}$ domains were mixed together to form the 359 complex and analyzed using XL-MS. We identified 6 local crosslink pairs consistent with 360 pairs observed in full-length DnaJB8 and the isolated JD 1-77 and CTD 170-232 samples (Fig. $3615 b)$. Importantly, we also reconstitute 4 intermolecular contacts between the $\mathrm{JD}_{1-77}$ and 362 CTD $_{170-232}$ observed in full length DnaJB8 experiments. However, an increased variance 363 in the crosslink profile may indicate that the missing proximal sequences help define the 364 proper architecture of the full-length DnaJB8 oligomers. Solution NMR-based chemical 365 shift perturbation (CSP) mapping was used to identify the JD1-77 surface that interacts 366 with the CTD $170-232$ (Fig. 5c, Supplementary Fig. 5b-c). Titration of increasing amounts of 367 unlabeled $\mathrm{CTD}_{170-232}$ into ${ }^{15} \mathrm{~N}$-labeled $\mathrm{JD}_{1-77}$ produced fast exchanging concentration368 dependent CSPs in a specific subset of peaks (Fig. 5d-e); 33 peaks were strongly 369 perturbed (>0.2 p.p.m.) and another 40 peaks were perturbed weakly (>0.1 p.p.m.). 370 Among the strongly perturbed peaks, 27 residues have solvent accessible side chains of 371 which fifteen are charged, with a notable overlap with the basic face in helix 2 that is 372 implicated in Hsp70 binding (Fig. 5f-h). Other residues that show strong perturbations are 373 basic and acidic residues that wrap around the exterior of the JD loop and helix 3, and 374 residues on the charged face of helix 4 . While a few other hydrophobic residues also 375 show strong perturbations, all are in close proximity to charged residues along each helix. 376 Given the small size of the $\mathrm{JD}_{1-77}$, it is likely that residues in the core behind the basic 377 surface involved in the interaction experience changes in chemical shift. 


\section{JD-CTD interaction competes with Hsp70 binding}

380 The recent X-ray structure of the DnaK:DnaJ complex revealed a conserved charge381 based interaction between the basic surfaces on the JD of DnaJ and an acidic surface on $382 \mathrm{DnaK}^{10}$. Using this complex as a template, we modeled the binding interface of the human $383 \mathrm{Hsp70}(\mathrm{HspA1A})^{41}$ and the JD of DnaJB842 (Fig. 6a-C). The basic surface on the DnaJB8 384 JD (Fig. 6b) contacts the conserved acidic surface on HspA1A (Fig. 6c, Supplementary 385 Fig. 6a-b). Thus, conserved electrostatic contacts are likely to play a key role in the 386 interaction between Hsp70 and Hsp40.

388 The conserved HspA1A-JD electrostatic contacts (Fig. 6b-c) that overlap with the JD-CTD 389 contact sites lead us to hypothesize that the observed JD-CTD interactions could interfere with Hsp70 binding. To test this hypothesis, we employed a competition experiment 391 leveraging our FP binding assay to discriminate the JD-CTD and JD-HspA1A complexes 392 (Fig. 6d). We determine a $0.257 \pm 0.029 \mu \mathrm{M}$ affinity for the JD:HspA1A interaction, 393 consistent with values in the literature ${ }^{43}$ and similar to the JD:CTD interaction (Fig. 6e, 394 black and green, respectively). Due to the size difference between HspA1A (70 kDa) and 395 the CTD $(8.7 \mathrm{kDa})$ their respective complexes with tagged JD plateau at different 396 polarization values (Supplementary Fig. 6c, black and green, respectively). Leveraging 397 this difference, we designed a binding experiment to measure the competition of HspA1A 398 and CTD binding to the JD. FITC-labeled JD was preincubated with $3 \mu \mathrm{M} \mathrm{CTD}$, followed 399 by a titration with HspA1A. The pre-titration FP signal was consistent with formation of 400 the JD-CTD complex, which persisted until HspA1A concentrations of 3.125uM when the 401 signal shifted to that of a JD-HspA1A complex (Supplementary Fig. 6c, purple). 402 Normalization of the data reveals a $\sim 25$-fold decrease in the apparent binding constant 403 between JD:HspA1A when preincubated with CTD (Fig. 6e). To further test the inhibitory 404 role of CTD on recruitment of Hsp70, we used XL-MS to measure the frequency of 405 HspA1A and JD contacts across a set of complexes formed between HspA1A and WT 406 DnaJB8, JD ${ }_{1-77}$, DnaJB8 $\mathrm{F}_{\rightarrow \mathrm{S}}$ and DnaJB8 $\triangle \mathrm{CTD}$ missing the CTD (Fig. 6f). Across three 407 experiments, we detected no crosslinks between the Hsp70 and the JD in WT DnaJB8 
408 and only 2 in DnaJB8 $\mathrm{F}_{\rightarrow \mathrm{S}}$ (Fig. 6g, Supplementary Data 1). In contrast, in the HspA1A:JD

40977 and HspA1A:DnaJB8 $\triangle$ CTD complexes, we identified 47 and 14 total crosslinks

410 between the JD and HspA1A, respectively (Fig. 6g). All identified pairs are consistent with

411 the structural model (Fig. 6h and Supplementary Fig. 6d). These data support that the

412 robust JD:CTD engagement seen in WT DnaJB8 and even the monomeric DnaJB $\mathrm{F}_{\mathrm{F} \rightarrow \mathrm{S}}$

413 (Supplementary Fig. 6e) prevents HspA1A interaction with the JD domain and deletion of

414 the CTD releases the inhibitory effect (Fig. $6 \mathrm{~g}$ ). Thus, the DnaJB8 JD uses a basic surface

415 to bind an internally encoded CTD via an acidic surface that directly inhibits HspA1A

416 binding.

DISCUSSION

\section{Modeling the shape of DnaJB8}

421 DnaJB8, like DnaJB6b, has the capacity to assemble into soluble oligomers. We used a 422 combination of protein engineering, solution scattering data and modeling to understand 423 the shapes of DnaJB8 in solution. Using our XL-MS data, we collapsed a DnaJB8 424 structural model around the JD:CTD interaction and thus obtained a structural model that 425 fit the average $\mathrm{Rh}$ of the monomer measured by DLS. Based on the fold of this monomeric 426 model, we hypothesized that aromatic amino acids in the central G/F and S/T domains 427 would be exposed and thus could mediate self-assembly into oligomers. Indeed, 428 mutagenesis of aromatic residues yielded a stable monomeric variant of DnaJB8 in 429 agreement with our collapsed structural model with engaged JD:CTD contacts. This is 430 further supported by good agreement between the $R_{h}$ of our collapsed structural model, 431 DLS data, and values derived from the Marsh and Forman-Kay model ${ }^{32}$. An intriguing 432 question relates to whether our models may also be applicable to DnaJB6b. At this time 433 a direct comparison is difficult given the known structural and functional differences of the 434 proteins and the lack of analogous experimental data, especially on the larger oligomers 435 of DnaJB6b. Our collective data highlights the power of our multipronged approach to 
436 derive the base unit of a DnaJB8 monomer, which employs exposure of aromatic residues

437 to mediate assembly through nonpolar surfaces into larger oligomers.

\section{Functional role of the CTD in DnaJB8}

440 We combined XL-MS and NMR in the solid and solution states to probe DnaJB8 inter441 domain interactions. One of the most striking features was a newly identified interaction 442 between the distal JD and CTD driven by electrostatics. This interaction was perturbed 443 by the addition of salt, but maintained following mutagenesis of aromatic amino acids in 444 the central domains. Since analysis of the isolated JD and CTD showed a reduced mutual 445 association, there nonetheless is a distinct role for the intervening domains in the JD-CTD 446 interaction. Our combined data show that the DnaJB8 S/T and G/F domains are not 447 behaving as "flexible linkers"17 and that their aromatic residues are central in the homo448 oligomerization process. On their own, both JD and CTD are surprisingly resistant to self449 assembly. These findings are distinct from published reports on DnaJB6b, where the CTD 450 appears to drive oligomerization, which may relate to sequence divergence in the six C451 terminal CTD residues between DnaJB8 and DnaJB6b ${ }^{17-18}$. Nonetheless, our modeled 452 CTD structure, featuring a pleated $\beta$-sheet topology absent of a hydrophobic core, is 453 identical to its recently reported DnaJB6b counterpart ${ }^{17}$. Interestingly, outside of inter454 strand hydrogen bonding and polar side-chain contacts, it is not clear what forces stabilize 455 this domain. This may explain the CTD heterogeneity (unlike the JD) seen by ssNMR. 456 The CTD topology resembles the charged $\beta$-sheet surface on Hsp70 that is known to 457 interact with the JD ${ }^{10}$. While our reconstitution of the JD:CTD interaction using isolated 458 domains indicates that the CTD alone can bind the JD, we cannot exclude that helix 5 459 can contribute to this interaction to regulate Hsp70 function. It is worth noting that lysine 460 residues in the DnaJB8 and DnaJB6b CTD can be acetylated and deacetylated (via 461 Histone Deacetylases (HDACs)) to modify these proteins' self-assembly and function, 462 which may involve changes in the Lys-mediated JD interactions ${ }^{16,20}{ }^{20}$ The CTD 463 architecture is conserved in a broader subset of B family member Hsp40s ${ }^{18}$. We speculate 464 that the CTD in these DnaJB family members similarly serves a regulatory role in which 
465 post-translational modifications could alter the affinity for the JD, and thus indirectly alters 466 oligomerization or Hsp70 recruitment.

Implications for Hsp70 recruitment and substrate binding.

469 Aside from suppressing protein aggregation on its own ${ }^{16,18,20}$, DnaJB8 also recruits $470 \mathrm{Hsp} 70$ for processing of bound substrates. Our current findings hint at an intriguing 471 possibility that auto-inhibitory interactions of the Hsp70-binding JDs within the DnaJB8 472 oligomer could be involved in substrate-binding-coupled Hsp70 recruitment. In the non473 stressed native state, DnaJB8 forms soluble oligomers in which the JD is engaged in 474 electrostatic interactions and thus not available for Hsp70 binding as supported by our 475 experiments (Fig. 6g; Fig. 7a). We hypothesize that substrate binding could allosterically 476 disrupt the JD-CTD interaction, exposing the Hsp70-binding HPD motif of the JD (Fig. 477 7b). This would enable the recruitment of Hsp70 to the loaded DnaJB8 protein. Aromatic478 driven oligomeric assembly of DnaJB8 may be related to the formation of liquid-liquid 479 phase separated assemblies in other proteins containing similar arrangements of 480 phenylalanine residues ${ }^{44}$. We propose that the more hydrophobic elements of the G/F 481 and $\mathrm{S} / \mathrm{T}$ domains form the oligomer core, with the CTD and JD remaining relatively 482 surface exposed. Thus, it may be possible to recruit Hsp70 to different DnaJB8 species. 483 Our data on the DnaJB8 $\mathrm{F}_{\rightarrow}$ s mutant illustrate that the JD:CTD interaction exists in the 484 monomeric base unit suggesting that this interaction is present across the polydisperse 485 distribution of DnaJB8 species. Although we as yet lack detailed information supporting 486 a substrate-triggered modulation of the JD:CTD interaction, our results offer some hints 487 toward a possible molecular mechanism for such a coupling. In in vivo and in vitro XL-MS 488 experiments, negatively charged residues in helix 5 in the G/F domain interact with both 489 the JD and CTD (Fig. 1). We also saw a change in JD:CTD affinity in the absence of the 490 central domains (Fig. 5). Finally, other studies on DnaJB6b have identified the S/T 491 domains as substrate binding domains ${ }^{16,20-21}$. Future mechanistic and structural studies 492 on DnaJB8 and other complex chaperones including DnaJB6b and their interactions with 493 substrates will reveal the interplay between oligomer dynamics, post-translational 
494 modifications, substrate binding, and recruitment of Hsp70.

\section{Materials and Methods}

\section{Sequence and structural analysis of DnaJB8, DnaJB6b and HspA1A}

499 Analysis of protein sequences (including the net charge per residue; NCPR) was 500 performed using Local CIDER ${ }^{45}$. An ensemble of 1000 HspA1A homology models was 501 produced using ab initio Rosetta using the DnaK (PDB:5NRO) conformation as a 502 template ${ }^{10}$. Briefly, the HspA1A sequence was aligned to the DnaK sequence to identify 503 regions with loop insertions and deletions. The HspA1A fragment library was produced 504 using the fragment picker. The lowest scoring model was used to produce a model of the 505 complex between HspA1A and the JD of DnaJB8. The structural images were produced 506 using PyMOL.

508 Cell biological and biochemical analysis of DnaJB8-Clover cell lines

509 The human DNAJB8 protein-coding sequence was cloned using Gibson assembly into a 510 modified FM5 lentiviral expression plasmid ${ }^{46}$ in which the $U b C$ promoter was replaced by 511 a CMV promoter, the linker sequence was replaced by "GSAGSAAGSGEF" and

512 the YFP was replaced by $m$ Clover3. The resulting gene produced a DNAJB8-mClover3 513 fusion protein. In parallel, we produced a construct that expresses the fluorescent protein 514 (mClover3) but lacks DnaJB8. Both plasmids we separately co-transfected into HEK293T 515 cells along with helper plasmids ( $\mathrm{PCMV}$-VSV-G and psPAX2) to produce lentivirus, which

516 was harvested after 48hrs and used to produce polyclonal cell lines that expressed either 517 DnaJB8-mClover3 or mClover3. For crosslinking experiments, cells from a confluent $51810 \mathrm{~cm}^{2}$ cell culture dish were pelleted and lysed using an insulin syringe in 1xPBS with $5191 \mathrm{mM}$ DTT, 1mM PMSF, 1x EDTA-free Protease Inhibitor Cocktail (Roche), and 1\% 520 Digitonin. After spinning at $1000 x$ for $10 \mathrm{~min}$., the lysate was recovered and incubated 521 with a polyhistidine tagged anti-GFP nanobody (plasmid encoding the nanobody was a 522 kind gift from Dr. Judith Frydman and purified as described previously ${ }^{24}$ ) for 2.5 hours at $5234^{\circ} \mathrm{C}$. The lysate was then incubated with $25 \mu \mathrm{L}$ NiNTA beads (Clontech) for 1 hour at $4^{\circ} \mathrm{C}$ 
524 for binding. The beads were washed five times with $300 \mu \mathrm{L} 1 \mathrm{xPBS}$. The buffer for each

525 wash was removed after pulse spinning the beads via centrifugation. The beads were

526 preincubated for 5 minutes at $37^{\circ} \mathrm{C}$ and a final concentration of $57 \mathrm{mM} \mathrm{ADH}$ and $36 \mathrm{mM}$

527 DMTMM were added to each sample. Following a one-minute incubation with chemical

528 crosslinkers, the reaction was quenched with $1 \mathrm{mM}$ ammonium bicarbonate. After another

529 pulse spin to remove the buffer, the beads were resuspended in elution buffer, (8M Urea,

$530 \quad 0.5 \mathrm{M}$ imidazole, $\mathrm{pH}$ 7.5). After a final pulse spin, the supernatant was retained and 531 analyzed by mass spectrometry.

532

\section{Cross-Linking Reagents}

534 All crosslinking reagents used are commercially available: ADH (Sigma-Aldrich), and 535 mixed light and deuterated ADH (ADH-h8/d8) (Creative Molecules). DMTMM (Sigma536 Aldrich). For all crosslinking experiments, stock solutions were made of each crosslinking 537 reagent. A 1:1 ADH-do/ADH-d s solution was made at $100 \mathrm{mg} / \mathrm{mL}$ in $1 \times \mathrm{xPS}$ pH 7.4 (Sigma538 Aldrich). DMTMM (Sigma-Aldrich) was prepared at a $120 \mathrm{mg} / \mathrm{mL}$ concentration in $1 \mathrm{xPBS}$ $539 \mathrm{pH} 7$.

540

\section{Cross-Linking Mass Spectrometry}

542 The ex-vivo purified DnaJB8 was dialyzed to remove excess imidazole, and transferred 543 into 1xPBS pH 7.4 buffer. For the full-length DnaJB8 experiments, lyophilized DnaJB8 544 was resuspended in either $1 \mathrm{xPBS}(150 \mathrm{mM})$ or $1 \mathrm{xPBS}(285 \mathrm{mM})$ to a concentration of $545100 \mu \mathrm{M}$. The $\mathrm{JD}_{1-77}$ and CTD $170-232$ constructs were purified into $1 \times \mathrm{xPS}$ buffer, and were 546 prepared for XL-MS experiments at $100 \mu \mathrm{M}$ each. $2 \mu \mathrm{M}$ HspA1A in $1 \mathrm{xPBS}$ pH 7.4 buffer 547 and mixed with either $40 \mu \mathrm{M}$ DnaJB8, $40 \mu \mathrm{M} \mathrm{JD}_{1-77}, 40 \mu \mathrm{M}$ DnaJB8 $\Delta \mathrm{CTD}$ and $40 \mu \mathrm{M}$ 548 DnaJB8 $\mathrm{F} \rightarrow \mathrm{S}$ for XLMS experiments and performed in triplicate. All samples were 549 incubated at $37^{\circ} \mathrm{C}$ while shaking at $350 \mathrm{rpm}$ for 30 minutes. Final concentrations of $57 \mathrm{mM}$ $550 \mathrm{ADH} \mathrm{d}_{0} / \mathrm{d}_{8}$ (Creative Molecules) and 36mM DMTMM (Sigma-Aldrich) were added to the 551 protein samples and incubated at $37^{\circ} \mathrm{C}$ with shaking at $350 \mathrm{rpm}$ for 30 minutes. The 552 reactions were quenched with $100 \mathrm{mM}$ ammonium bicarbonate and incubated at $37^{\circ} \mathrm{C}$ for 
$55330 \mathrm{~min}$. Samples were lyophilized and resuspended in 8M urea. Samples were reduced

554 with $2.5 \mathrm{mM}$ TCEP incubated at $37^{\circ} \mathrm{C}$ for $30 \mathrm{~min}$ followed by alkylation with $5 \mathrm{mM}$

555 iodoacetimide for 30 minutes in the dark. Samples were diluted to $1 \mathrm{M}$ urea using a stock

556 of $50 \mathrm{mM}$ ammonium bicarbonate and trypsin (Promega) was added at a 1:50 enzyme-to-

557 substrate ratio and incubated overnight at $37^{\circ} \mathrm{C}$ shaking at $600 \mathrm{rpm} .2 \%(\mathrm{v} / \mathrm{v})$ formic acid 558 was added to acidify the samples following overnight digestion. All samples were run on 559 reverse phase Sep-Pak tC18 cartridges (Waters) eluted in $50 \%$ acetonitrile with $0.1 \%$ 560 formic acid. $10 \mu \mathrm{L}$ of the purified peptide fractions was injected for LC-MS/MS analysis on 561 an Eksigent 1D-NanoLC-Ultra HPLC system coupled to a Thermo Orbitrap Fusion Tribrid 562 system. Peptides were separated on self-packed New Objective PicoFrit columns $(11 \mathrm{~cm}$ $563 \times 0.075 \mathrm{~mm}$ I.D.) containing Magic C18 material (Michrom, $3 \mu \mathrm{m}$ particle size, $200 \AA$ pore 564 size) at a flow rate of $300 \mathrm{~nL} / \mathrm{min}$ using the following gradient. $0-5 \mathrm{~min}=5 \% \mathrm{~B}, 5-95 \mathrm{~min}=$ 565 5-35\%B, 95-97min $=35-95 \% \mathrm{~B}$ and 97-107min $=95 \% \mathrm{~B}$, where $\mathrm{A}=$ 566 (water/acetonitrile/formic acid, 97:3:0.1) and $B=$ (acetonitrile/water/formic acid, 97:3:0.1).

567 The mass spectrometer was operated in data dependent mode by selecting the five most 568 abundant precursor ions ( $\mathrm{m} / \mathrm{z}$ 350-1600, charge state $3+$ and above) from a preview scan 569 and subjecting them to collision-induced dissociation (normalized collision energy $=35 \%$, $57030 \mathrm{~ms}$ activation). Fragment ions were detected at low resolution in the linear ion trap.

571 Dynamic exclusion was enabled (repeat count 1, exclusion duration 30sec).

572

\section{Analysis of Mass Spectrometry Results}

574 All mass spectrometry experiments were carried out on an orbitrap Fusion Lumos Tribrid 575 instrument available through the UTSW proteomics core facility. Each Thermo .raw file 576 was converted to .mzXML format for analysis using an in-house installation of $x$ Quest ${ }^{47}$. 577 Score thresholds were set through xProphet $^{47}$, which uses a target/decoy model. The 578 search parameters were set as follows. For grouping light and heavy scans (hydrazide 579 crosslinks only): Precursor mass difference for isotope-labeled hydrazides $=8.05021 \mathrm{Da}$ 580 for $\mathrm{ADH}-\mathrm{d}_{0} / \mathrm{d}_{8}$; maximum retention time difference for light/heavy pairs $=2.5 \mathrm{~min}$.

581 Maximum number of missed cleavages $=2$, peptide length $=5-50$ residues, fixed 
582 modifications $=$ carbamidomethyl-Cys $($ mass shift $=57.02146 \mathrm{Da}$ ), mass shift of light 583 crosslinker $=138.09055$, mass shift of monolinks $=156.1011 \mathrm{Da}, \mathrm{MS}^{1}$ tolerance $=15$

$584 \mathrm{ppm}$, and $\mathrm{MS}^{2}$ tolerance $=0.2 \mathrm{Da}$ for common ions and 0.3 Da for crosslink ions; search 585 in enumeration mode. For zero-length crosslink search: maximum number of missed 586 cleavages $=2$, peptide length $=5-50$ residues, fixed modifications carbamidomethyl-Cys 587 (mass shift $=57.02146 \mathrm{Da}$ ), mass shift of crosslinker $=-18.010595 \mathrm{Da}$, no monolink mass 588 specified, $\mathrm{MS}^{1}$ tolerance $=15 \mathrm{ppm}$, and $\mathrm{MS}^{2}$ tolerance $=0.2 \mathrm{Da}$ for common ions and 0.3 589 Da for crosslink ions; search in enumeration mode. The FDRs of all in vitro experiments 590 range from 0.05 to 0.33 .

591

592 Western blot analysis

$59310 \mu \mathrm{L}$ aliquots of the HEK control, Clover, and DnaJB8-Clover cell-lines were removed 594 from the elution and loaded onto a $4-12 \%$ Bis-Tris SDS-PAGE gel for western blotting. 595 Upon running the gel to completion, the gel transferred onto a transfer membrane soaked 596 in Novoblot transfer buffer. Following the transfer, the membrane was soaked in milk 597 blocking buffer for 1 hour at room temperature. For immunolabelling, we added 1:2000 598 dilution of polyclonal Anti-GFP (rabbit) (Rockland; 600-401-215; 35460) or Anti-DnaJB8 599 (rabbit) (abcam; ab235546; GR3229943-2) in milk and incubated the membrane shaking 600 at room temperature for 2 hours. The primary antibody solution was dumped and the 601 membrane washed three times for 10 minutes each with 1xTBST before adding the 602 polyclonal Anti-Rabbit IgG Peroxidase (GE Healthcare; NA9340V; 16908235) at a 1:5000 603 dilution in milk. The membrane was incubated with secondary antibody at room 604 temperature for 1 hour before removing the antibody solution. The membrane was 605 washed 3 times in 5-minute intervals with 1xTBST and finally one 5-minute wash with 606 1xTBS. The membrane was soaked in $1 \mathrm{~mL}$ of Luminol enhancer and peroxide solution 607 for 1 minute before imaging.

608 609

\section{In cell analysis of DnaJB8-Clover and Clover cell lines}


610 HEK293T cells were treated with $1 x$ and $3 x$ amounts of lentivirus expressing either

611 DnaJB8-mClover3 or mClover alone were plated at 300,000 cells per well in media 612 (10\%FBS, 1\%Pen/Strep, 1\%GlutaMax in DMEM) in a 6-well glass bottom plate (Cellvis, 613 P06-1.5-N). After 30 hours, cells were stained with Hoescht33342 at a final concentration 614 of $2 \mathrm{ug} / \mathrm{mL}$ in cell media for 30 minutes at $37 \mathrm{C}$ and $5 \% \mathrm{CO} 2$. The plate was placed on an 615 IN Cell 6000 Analyzer (GE Healthcare) with a heated stage and fifty fields of view were 616 imaged under DAPI and FITC channels at 60X magnification (Nikon 60X/0.95, Plan Apo, 617 Corr Collar 0.11-0.23, CFI/60 lambda). Images were exported as TIFF files for 618 downstream analysis. DnaJB8-mClover3, mClover3, and wild-type HEK293 cells were 619 plated and imaged in triplicates. Total cell counting was done using the CellProfiler v3.0 620 software 48 by selecting for DAPI (total cells in acquired images) and mClover3 (total 621 expressing cells in acquired images). Punctae-containing cells were counted manually by 622 two different observers and the data reported as the average with standard deviation 623 between both observers. Expression of Clover and DnaJB8-Clover in 1x and 3x cell lines 624 was quantified from Western blot analysis and by fluorescence intensity of Clover 625 quantified using ImageJ49.

\section{Recombinant expression and purification of DnaJB8 and DnaJB8 $\Delta$ CTD}

628 The vector used for DnaJB8 expression was a pET-29b vector containing the gene for 629 human DnaJB8, a T7 promoter to activate DnaJB8 expression, a His tag region at the 630 end of the gene, and a gene for kanamycin resistance. The DnaJB8 $\triangle$ CTD fragment was 631 cloned into pet29b using Gibson assembly. The same protocol was used to express and 632 purify the WT DnaJB8 and DnaJB8 $\triangle$ CTD proteins. The vector constructs were 633 transformed into E. coli BL-21 (DE3) cells and plated onto 2xLB plates containing $6340.05 \mathrm{mg} / \mathrm{mL}$ Kanamycin. $12 \mathrm{~mL}$ of $2 x L B, 0.05 \mathrm{mg} / \mathrm{mL}$ Kanamycin were prepared and 635 inoculated with a single colony from the plate. This small culture was incubated overnight 636 at $37^{\circ} \mathrm{C}$ while shaking at $220 \mathrm{rpm}$. In the morning, the $12 \mathrm{~mL}$ culture was added to $1 \mathrm{~L}$ of $6372 x L B$ supplemented with $0.05 \mathrm{mg} / \mathrm{mL}$ Kanomycin and incubated at $37^{\circ} \mathrm{C}$ while shaking at 638 220rpm. Once OD600 reached 0.6-0.8 A.U., 1mL of 1M IPTG was added to induce 
639 DnaJB8 expression. After incubation for an additional 4 hours, the cells were harvested 640 by spinning down the culture at 4,000g for 20 minutes. The resulting cell pellet was 641 resuspended in $25 \mathrm{~mL} 1 \mathrm{xPBS}$ and $1 \mathrm{mM}$ PMSF in preparation for insoluble fraction 642 separation. The resuspended cells were sonicated at $30 \%$ power, $5 \mathrm{X}$ pulse for 10 minutes 643 using an Omni Sonic Ruptor 4000 (Omni International). The lysed cells were pelleted at $64410,000 \mathrm{~g}$ for 30 minutes and the supernatant was discarded. The insoluble pellet was 645 rinsed with 1xPBS, 0.75\%Tween-20 and again pelleted at 10,000g for 30 minutes.

646 The insoluble cell pellet was resuspended in $50 \mathrm{~mL}$ lysis buffer $(8 \mathrm{M}$ Guanidinium $\mathrm{HCl}$, 647 50mM HEPES, 20mM Imidazole, 1mM DTT pH 7.5) and sonicated at 30\% power, 3X 648 pulse for 1 minute to solubilize the DnaJB8 from the insoluble pellet. After a 30-minute 649 incubation at room temperature, the cellular debris was pelleted at $15,000 \mathrm{~g}$ for 30

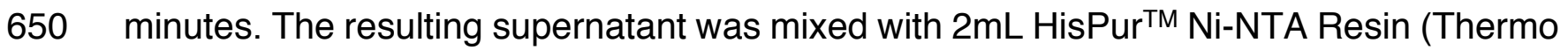
651 Scientific) for 1 hour before being loaded onto a gravity column. The column was washed 652 with an additional $50 \mathrm{~mL}$ of lysis buffer, followed by $50 \mathrm{~mL}$ of a second wash buffer $(50 \mathrm{mM}$ 653 HEPES, 20mM Imidazole, 1mM DTT pH 7.5 in $\mathrm{H}_{2} \mathrm{O}$ ). The protein was eluted with $30 \mathrm{~mL}$ 654 of elution buffer (50mM HEPES, 500mM Imidazole, $1 \mathrm{mM} \mathrm{DTT} \mathrm{pH} 7.5$ in $\mathrm{H}_{2} \mathrm{O}$ ) and 655 collected in $2 \mathrm{~mL}$ fractions. After selecting for fractions with high purity, the DnaJB8 656 solution was loaded into 3.5kDa cutoff Biotech CE Dialysis Tubing (Spectrum Labs) and 657 dialyzed overnight at $4 \stackrel{\circ}{-} \mathrm{C}$ in $50 \mathrm{mM}$ ammonium formate to minimize assembly. The protein 658 was then lyophilized and stored at $-80^{\circ} \mathrm{C}$ for future use.

660 Dynamic Light Scattering

661 All samples were prepared at $1.2 \mathrm{mg} / \mathrm{mL}$ in $1 \mathrm{xPBS}, 1 \mathrm{mM}$ DTT, $\mathrm{pH}$ 7.4. All protein samples 662 were filtered through a $0.22 \mu \mathrm{m}$ PES sterile filter and loaded in triplicate onto a 384 well 663 clear flat-bottom plate. The plate was loaded into a Wyatt DynaPro Plate Reader III and 664 set to run continuously at room temperature at a scanning rate of 1 scan per 15 minutes, 665 with 1 scan composed of 10 acquisitions. The data were analyzed using the Wyatt 666 Dynamics software version 7.8.2.18. Light scattering results were filtered by Sum of 667 Squares (SOS) $<20$ to eliminate statistical outlier acquisitions within each scan. For 
668 DnaJB8 in 1xPBS(150mM) buffer, one of the triplicates contains partial data due to high 669 SOS values. This is a result of increasing polydispersity and heterogeneity, which is 670 consistent with oligomers of that size. $R_{h}$ of observed particles for three time points (0h, 671 10h, and $20 \mathrm{~h}$ ) were reported as histograms as a function of mass $\%$. The mass $\%$ 672 contribution of smaller particles in the full-length DnaJB8 runs in $1 \times P B S(150 \mathrm{mM})$ and 673 (285mM) buffer were reported as a function of mass\% over time using the SOS filter and 674 a size filter of $<10 \mathrm{~nm}$. Data for DnaJB8 $\mathrm{F}_{\rightarrow \mathrm{S}}$, JD ${ }_{1-77}$ and $\mathrm{CTD}_{170-232}$ was reported as a 675 function of $R_{h}$ over time with the SOS filter applied.

\section{Modeling of full length DnaJB8 using Rosetta and XL-MS restraints}

678 Given the globular conformations of the JD and CTD we considered how the crosslinks 679 identified for the full-length protein could guide the JD-CTD interaction. Using Rosetta we 680 assembled a monomeric conformation leveraging the JD and CTD conformations while 681 keeping the G/F and $\mathrm{S} / \mathrm{T}$ regions fully expanded. This starting model was then used in a 682 relax protocol in conjunction with crosslinks (Supplementary Data 1) as constraints to 683 produce an ensemble of 1000 collapsed conformations. A representative low scoring 684 model was selected for further analysis. For acid-acid and acid-lysine contacts 21 and 16 685 A distance thresholds were used as restraints. The HYDROPRO50 software was used to 686 calculate radii of hydration from structural models.

\section{Conservation mapping}

689 DnaJB8 homolog sequences were identified using Blast ${ }^{51-52}$ and the sequences were 690 aligned using Clustal Omega ${ }^{53}$. The protein sequence alignment and structure of DnaJB8 691 JD (PDBID 2DMX) were used as input in $\mathrm{Al} 2 \mathrm{Co}^{54}$ to map the conservation onto the 692 structural models. The conservation was mapped onto the models in PyMOL.

693

\section{Coevolutionary variation analysis}


695 The GREMLIN software $29-31$ was used to identify covarying amino acid pairs from a

696 DnaJB8 protein sequence alignment. A probability of 0.7 was used to threshold the data

697 to identify amino acids with strong coupling.

698

\section{Solid state NMR analysis}

700 Chaperone oligomers were prepared in PBS buffer with different $\mathrm{NaCl}$ concentrations.

701 Lyophilized DnaJB8 uniformly labeled with ${ }^{13} \mathrm{C}$ and ${ }^{15} \mathrm{~N}\left(\mathrm{U}-{ }^{13} \mathrm{C},{ }^{15} \mathrm{~N}\right)$ was re-suspended in

$7021 \mathrm{ml}$ of PBS buffer in which the final $\mathrm{NaCl}$ concentration was $100 \mathrm{mM}$ or $285 \mathrm{mM}$, 703 respectively, for the two samples measured by MAS NMR. Each sample was packed into

704 a 3.2mm MAS NMR rotor (Bruker Biospin) by sedimentation, using a previously described 705 ultracentrifugal packing tool ${ }^{55}$. Sedimentation was done at 175,000 x g force in an Optima

706 L-100 XP Ultracentrifuge using a SW-32 Ti centrifuge rotor for 1 hour. Subsequently, 707 excess of the supernatant fluid was removed. Sample tubes were washed with another $7081 \mathrm{~mL}$ buffer solution after which a second packing step using the same parameters was 709 performed. Finally, the supernatant was removed, spacers were placed on the top of the 710 hydrated sedimented protein oligomers, and rotors were closed with the drive cap, and 711 sealed with a small amount of epoxy to avoid sample dehydration.

713 Experiments were performed on Bruker $600 \mathrm{MHz}$ and $750 \mathrm{MHz}$ spectrometers, at $277 \mathrm{~K}$ 714 temperature using triple-channel (HCN) 3.2mm MAS EFree probes. All experiments were 715 done using TPPM ${ }^{56}$ proton decoupling of $83 \mathrm{kHz}$ during acquisition. Single pulse 716 excitation (SPE) measurements were performed using a $6 \mu \mathrm{s} 90^{\circ}$ pulse on ${ }^{13} \mathrm{C}$, 3s recycle 717 delay, $1 \mathrm{k}$ scans and 1D Cross-Polarization experiments (CP) were performed using a $7183.1 \mu \mathrm{s} 90^{\circ} 1 \mathrm{H}$ pulse, $2 \mathrm{~ms}$ contact time, recycle delay of $3 \mathrm{~s}$, $1 \mathrm{k}$ scans. 1D refocused INEPT 719 experiments were done at using $3 \mu \mathrm{s} 90^{\circ}$ proton pulse, $4 \mu \mathrm{s} 90^{\circ}$ pulse on ${ }^{13} \mathrm{C}$, recycle delay 720 of $2 \mathrm{~s}$ and $1 \mathrm{k}$ scans. The ${ }^{13} \mathrm{C}-{ }^{13} \mathrm{C} 2 \mathrm{D}$ CP-DARR experiments were performed using $25 \mathrm{~ms}$ 721 mixing time, $0.4 \mathrm{~ms}$ contact time, $3.1 \mu \mathrm{s} 90^{\circ}$ proton pulse, $6 \mu \mathrm{s} 90^{\circ}$ pulse on ${ }^{13} \mathrm{C}$, recycle 722 delay of $2.8 \mathrm{~s}, 128$ scans. The $2 \mathrm{D}$ NCA experiment was performed using $3 \mu \mathrm{s} 90^{\circ}$ proton 723 pulse, $900 \mu \mathrm{s}$ and $3.25 \mathrm{~ms}{ }^{1} \mathrm{H}-{ }^{13} \mathrm{C}$ and ${ }^{13} \mathrm{C}-15 \mathrm{~N}$ contact times respectively, $8 \mu \mathrm{s} 180^{\circ}$ pulse 
724 on ${ }^{15} \mathrm{~N}$, recycle delay of 2 s, 576 scans. The amino acid type and secondary structure were 725 predicted using the PLUQ program ${ }^{38}$ applied to the chemical shifts in the $2 \mathrm{D}{ }^{13} \mathrm{C}-{ }^{13} \mathrm{C}$ 726 spectrum. Linewidth analysis was done using the UCSF Sparky NMR analysis program ${ }^{57 .}$

727 Spectral acquisitions were done with Bruker Topspin software and processing was done 728 with the NMRpipe and Sparky software packages ${ }^{57-59}$.

\section{Simulations and synthetic NMR spectra}

731 The structure of the DnaJB8 JD in solution was determined previously using solution $732 \mathrm{NMR}^{42}$, allowing us also to generate a synthetic ${ }^{13} \mathrm{C}-{ }^{13} \mathrm{C} 2 \mathrm{D}$ spectrum using the 733 corresponding solution NMR chemical shifts from the BMRB (entry 11417). To simulate

734 approximate 2D NMR spectra of the other three domains, we made use of the results of 735 MD simulations of full length DnaJB8. The starting DnaJB8 conformation was produced 736 using ROSETTA with a fully expanded conformation of the G/F and S/T domains while 737 keeping the JD and CTD in the folded conformations. MD simulations were prepared 738 using Maestro ${ }^{60-61}$ and carried out in Desmond running the amber99 forcefield. Estimated 739 chemical shifts of the resulting structural models were generated using the SPARTA+ 740 package ${ }^{39}$.

742 Recombinant Expression and Purification of DnaJB8 $\mathrm{F} \rightarrow \mathrm{s}$, J Domain, and C-Terminal 743 Domain

744 Both vector constructs containing DnaJB8 JD and CTD respectively were cloned into 745 pET-29b using Gibson assembly. The DnaJB8 $\mathrm{F}_{\rightarrow}$ s construct was purchased from 746 Genscript and cloned into pET-29b. These vectors were transformed into E. coli BL-21 747 (DE3) cells and plated onto $2 x L B$ plates with $0.05 \mathrm{mg} / \mathrm{mL}$ Kanamycin. $12 \mathrm{~mL}$ of $2 x L B$ with $748 \quad 0.05 \mathrm{mg} / \mathrm{mL}$ Kanamycin were prepared and inoculated with a single colony from each 749 plate. These small cultures were incubated overnight at $37^{\circ} \mathrm{C}$ while shaking at $220 \mathrm{rpm}$. 750 In the morning, the $12 \mathrm{~mL}$ culture was added to $1 \mathrm{~L}$ of $2 x \mathrm{LB}$ supplemented with $0.05 \mathrm{mg} / \mathrm{mL}$ 751 Kanamycin and incubated at $37^{\circ} \mathrm{C}$ while shaking at 220rpm. Once OD600 reached 0.67520.8 A.U., $1 \mathrm{~mL}$ of $1 \mathrm{M}$ IPTG was added to induce protein expression. After incubation for 
753 an additional 4 hours, the cells were harvested by spinning down the culture at 4,000g for

75420 minutes. For preparing ${ }^{15} \mathrm{~N}$ JD, a single colony was inoculated into $10 \mathrm{~mL} 2 \times \mathrm{LB}$ 755 supplemented with $0.05 \mathrm{mg} / \mathrm{mL}$ Kanamycin and incubated for 7-8 hours at $37^{\circ} \mathrm{C}$ while 756 shaking at 220rpm. The $10 \mathrm{~mL}$ culture was then mixed into $100 \mathrm{~mL}$ of M9 minimal media 757 (42mM Na $2 \mathrm{HPO}_{4}, 22 \mathrm{mM} \mathrm{KH}_{2} \mathrm{PO}_{4}, 8.5 \mathrm{mM} \mathrm{NaCl}, 0.1 \mathrm{mM} \mathrm{CaCl}, 2 \mathrm{mM} \mathrm{Mg}_{2} \mathrm{SO}_{4}, 1 \mathrm{E}-4 \%$ 758 Thiamine, $0.4 \%$ Glucose, $187 \mathrm{mM} \mathrm{NH}_{4} \mathrm{Cl}, 0.05 \mathrm{mg} / \mathrm{mL}$ Kanamycin) and incubated 759 overnight at $37^{\circ} \mathrm{C}$ while shaking at 220rpm. In the morning of the following day, the cells 760 were spun down at 2,000g for 10 minutes and resuspended in $20 \mathrm{mLs}$ of $\mathrm{M9}$ minimal media 761 containing $15 \mathrm{~N}$ labeled $\mathrm{NH}_{4} \mathrm{Cl}$ in place of the unlabeled molecule. This was immediately 762 added to $1 \mathrm{~L}$ of $\mathrm{M} 9$ minimal media with ${ }^{15} \mathrm{~N}$ labeled $\mathrm{NH}_{4} \mathrm{Cl}$ and allowed to incubate at $37^{\circ} \mathrm{C}$ 763 shaking at 220rpm. Once OD600 reached 0.6-0.8 A.U., 1mL of 1M IPTG was added to 764 induce protein expression. After incubation for an additional 4 hours, the cells were 765 harvested by spinning down the culture at 4,000g for 20 minutes. The cell pellets were 766 resuspended in $20 \mathrm{~mL}$ of soluble wash buffer (SWB) $\left(50 \mathrm{mM} \mathrm{KPO}_{4}, 300 \mathrm{mM} \mathrm{NaCl}, 10 \%\right.$ 767 glycerol, 1mM PMSF, 10mM BME, pH 8) and sonicated at 30\% power, 5X pulse for 10 768 minutes using an Omni Sonic Ruptor 4000 (Omni International). After incubation at room 769 temperature for 1 hour, the cell lysate was spun down at 15,000g for 30 minutes to 770 separate the soluble supernatant from the insoluble pellet. The supernatant was mixed 771 with $2 \mathrm{~mL}$ TALON ${ }^{\circ}$ Metal Affinity Resin (Clontech) and incubated shaking at $4^{\circ} \mathrm{C}$ for 1 772 hour. The protein-resin slurry was loaded onto a gravity column and washed with an 773 additional $40 \mathrm{~mL}$ of SWB. This was followed by subsequent washes: $20 \mathrm{~mL}$ SWB with $0.5 \%$ 774 tritonX-100, 20mL SWB with adjusted $700 \mathrm{mM} \mathrm{NaCl}, 20 \mathrm{~mL}$ SWB with $0.1 \mathrm{mM}$ ATP and $7755 \mathrm{mM} \mathrm{MgCl}_{2}$, and an additional $40 \mathrm{~mL}$ of SWB. The protein was eluted with $16 \mathrm{~mL}$ SWB 776 with $200 \mathrm{mM}$ Imidazole into $2 \mathrm{~mL}$ fractions. After selecting for fractions with high purity, the 777 protein solution was loaded into 3.5kDa cutoff Biotech CE Dialysis Tubing (Spectrum 778 Labs) and dialyzed overnight at $4^{\circ} \mathrm{C}$ in 1 XPBS to restore native folding. Both domain 779 constructs were further enriched by running on a GE Superdex 200 Increase 10/300 780 column in 1xPBS $1 \mathrm{mM}$ DTT $\mathrm{pH}$ 7. The protein was aliquoted and flash frozen in liquid 781 nitrogen and stored at $-80^{\circ} \mathrm{C}$ for future use. 


\section{SEC-MALS}

784 DnaJB8 $\mathrm{F}_{\rightarrow} \mathrm{S}, \mathrm{JD}_{1-77}$, and $\mathrm{CTD}_{170-232}$ constructs at a concentration of $5.1 \mathrm{mg} / \mathrm{mL}, 5.0$ $785 \mathrm{mg} / \mathrm{mL}$, and $6.6 \mathrm{mg} / \mathrm{mL}$, respectively, in $1 \times$ PBS were filtered through a $0.1 \mu \mathrm{m}$ filter to 786 remove larger impurities. Each sample was further filtered using a $0.22 \mu \mathrm{m}$ centrifugal 787 filter before $100 \mu \mathrm{L}$ was applied to a Superdex 200 Increase 10/300 column equilibrated 788 in 1xPBS. The column was in line with a Shimadzu UV detector, a Wyatt TREOS II light789 scattering detector, and a Wyatt Optilab tREX differential-refractive-index detector. The 790 flow rate was $0.5 \mathrm{~mL} / \mathrm{min}$. The data were analyzed with Wyatt's ASTRA software version 791 7.1.0.29. SEDFIT 62 was used to calculate the $\mathrm{dn} / \mathrm{dc}$ of the protein.

\section{Circular Dichroism}

794 Recombinant CTD $_{170-232}$ domain constructs were transferred into $10 \mathrm{mM} \mathrm{NaPO}_{4}, 150 \mathrm{mM}$ $795 \mathrm{NaF}, \mathrm{pH} 7.4$ buffer at a concentration of $40 \mu \mathrm{M}$. The experiment was run using a Jasco J796815 Circular Dichroism instrument with a PMT detector using a $10 \mathrm{~mm}$ quartz cuvette. 6 797 accumulations were taken at a speed of $50 \mathrm{~nm} / \mathrm{min}$ along the UV spectrum from $190 \mathrm{~nm}$ 798 to $300 \mathrm{~nm}$. Spectra analysis was done using the BeStSel online software ${ }^{63-64}$ to determine 799 secondary structural composition.

800

\section{CTD model generation}

802 Fragment libraries for the CTD sequence were generated using the Robetta server. 5000 803 models were produced using the $a b$ initio protocol and clustered to identify unique 804 conformations. Lowest scoring models from the top clusters showed high structural 805 similarity. The identified crosslinks from the CTD in full length DnaJB8 or isolated CTD 806 were evaluated for consistency with each model in the ensemble. For acid-acid and acid807 lysine crosslinks a distance threshold of 21 and $16 \AA$, respectively, was considered as 808 satisfied and consistent with the chemistry. Distances were calculated using a custom 809 script in MATLAB ver.R2019a and the crosslink pairs were visualized using PyMOL. The 810 HYDROPRO ${ }^{50}$ software was used to calculate radii of hydration from structural models. 
812 Fluorescence polarization

$813 \mathrm{JD}_{1-77}$ was labelled with 10x FITC-maleimide (sigma) in 1xPBS 1mM TCEP for 2 hours at

814 room temperature. The reaction was quenched and the excess dye was removed using

815 a Zeba spin desalting column (Thermo). For all experiments, 0.2 $\mu \mathrm{M} \mathrm{JD}_{1-77}$ was incubated

816 in triplicate with a titration gradient of $\mathrm{Hsp} 70(150 \mu \mathrm{M}-0 \mu \mathrm{M})$ or CTD $170-232(150 \mu \mathrm{M}-0 \mu \mathrm{M})$ in

817 1xPBS, 1mM TCEP, pH 7.4. For competition experiments, labelled JD 1-77 was mixed with

$8183.125 \mu \mathrm{M}$ CTD in triplicate and incubated at room temperature for 1 hour before adding a

819 titration gradient of $\mathrm{Hsp} 70(150 \mu \mathrm{M}-0 \mu \mathrm{M})$. Fluorescence polarization readings were taken

820 with excitation at $494 \mathrm{~nm}$ and emission at $525 \mathrm{~nm}$. The data were fit to a one site specific

821 binding model using GraphPad Prism 7.04.

822

823 Microscale Thermophoresis

824 The MST binding experiments were performed on Nanotemper Monolith NT.115 in the

825 Molecular Biophysics Resource core at UTSW and analyzed with a standard protocol ${ }^{65}$.

826 Binding measurements were performed in triplicate. The JD 1-77 was labeled with FITC-

827 maleimide (sigma) and 200nM JD1-77-FITC was titrated by a serial 2-fold dilution of

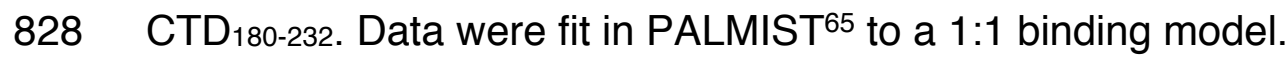

829

830

831 Solution NMR with ${ }^{15} \mathrm{~N}$-labeled JD and CTD

832 The $300 \mu \mathrm{M}{ }^{15} \mathrm{~N}$-labeled JD was exchanged into $20 \mathrm{mM}$ Tris $100 \mathrm{mM} \mathrm{NaCl} 1 \mathrm{mM}$ DTT pH 7 833 buffer in preparation for solution NMR. Each HSQC run was done for 4 hours at 1 834 scans/min with the temperature fixed at 299K. After each run, unlabeled CTD was titrated 835 into the sample at 1:1,1:3, and 1:6 ratios sequentially. All scans were collected on an 836 Agilent DD2 600MHz instrument at the UT Southwestern Biomolecular NMR Facility.

837 Each spectrum was converted into a readable format and phase corrected using 838 NMRPipe ${ }^{58}$. Peak assignments were based on the deposited information from BMRB 839 (11417). The software Sparky ${ }^{57,59}$ was used to analyze the peak shifts across all spectra. 


\section{Recombinant expression and purification of HspA1A}

843 HspA1A gene was cloned into the pMCSG7 plasmid66 and transformed into BL-21 (DE3)

844 cells and plated onto $2 x \mathrm{LB}$ plates with $0.1 \mathrm{mg} / \mathrm{mL}$ ampicillin. $12 \mathrm{~mL}$ of $2 x \mathrm{LB}$ with $0.1 \mathrm{mg} / \mathrm{mL}$

845 ampicillin were prepared and inoculated with a single colony from each plate. These small

846 cultures were incubated overnight at $37^{\circ} \mathrm{C}$ while shaking at $220 \mathrm{rpm}$. In the morning, the

$84712 \mathrm{~mL}$ culture was added to $1 \mathrm{~L}$ of $2 x \mathrm{xB}$ supplemented with $0.1 \mathrm{mg} / \mathrm{mL}$ ampicillin and 848 incubated at $37^{\circ} \mathrm{C}$ while shaking at $220 \mathrm{rpm}$. Once OD600 reached 0.6-0.8 A.U., $1 \mathrm{~mL}$ of 849 1M IPTG was added to induce protein expression. The cells continued to incubate 850 overnight at $12^{\circ} \mathrm{C}$ shaking at 220rpm. After incubation, the cells were lysed using a 851 PandaPlus 2000 homogenizer (GEA) by pressing the cells with 10,000p.p.m pressure. 852 The lysate was spun at $15,000 x g$ for 45 minutes to remove insoluble cell components, 853 and the resulting supernatant was mixed with $2 \mathrm{~mL}$ TALON® Metal Affinity Resin 854 (Clontech) and incubated at $4^{\circ} \mathrm{C}$ for 1 hour. The slurry was spun down at $700 \times \mathrm{xg}$ for 2 min 855 to remove the majority of the buffer and the beads were added onto a gravity column. The 856 beads were washed with $6 \mathrm{CV}$ of wash buffer $(50 \mathrm{mM}$ Tris, $500 \mathrm{mM} \mathrm{NaCl}, 10 \mathrm{mM}$ imidazole, $8575 \mathrm{mM} \beta M E, p H 8)$ and eluted with $5 \mathrm{~mL}$ of elution buffer $(50 \mathrm{mM}$ Tris, $500 \mathrm{mM} \mathrm{NaCl}, 300 \mathrm{mM}$ 858 imidazole, $5 \mathrm{mM}$ ßME, pH 8). HspA1 A containing fractions were confirmed by SDS-PAGE 859 and pooled together for desalting. Desalting/buffer exchange was performed using a PD86010 desalting column (GE Healthcare), where HspA1A fractions were transferred into 861 anion-exchange wash buffer (50mM Tris, $20 \mathrm{mM} \mathrm{NaCl}, 1 \mathrm{mM}$ DTT, pH 8.75). The protein 862 was loaded onto a HiTrap Q HP anion exchange column (GE Healthcare) and eluted 863 across a gradient of anion-exchange elution buffer (50mM Tris, $1 \mathrm{M} \mathrm{NaCl}, 1 \mathrm{mM}$ DTT, pH 864 8.75). HspA1A-containing fractions were once again combined and loaded onto a 865 Superdex ${ }^{\mathrm{TM}} 200$ Increase 10/300 GL (GE Life Sciences) size exclusion column, where 866 HspA1A was further purified and transferred into 1xPBS, 1mM DTT, pH 7.4 buffer for all 867 subsequent experiments. 
870 This work was supported by grants from the Welch Foundation and the Effie Marie Cain 871 Endowed Scholarship (L.A.J.) and NIGMS R01 GM112678 (P.V.D.W.). We appreciate

872 the help of the Molecular Biophysics Resource core, Structural Biology Laboratory, 873 Biomolecular Nuclear Magnetic Resonance Facility, and Proteomics Core Facility at the 874 University of Texas Southwestern Medical Center. The $750 \mathrm{MHz}$ ssNMR instrument at 875 the University of Pittsburgh was acquired with funding from NIH grant 10 OD012213-01.

\section{Author Contributions}

878 B.D.R., I.M., P.V.D.W., and L.A.J. conceived and designed the overall study. B.D.R. performed in 879 vitro protein binding assays, cell models, crosslink mass spectrometry, and ROSETTA 880 simulations. I.M. and P.V.D.W. performed ssNMR experiments and analyzed the data. S.B. 881 performed DnaJB8 CTD experiments and ROSETTA simulations. J.V.A produced mammalian 882 cell lines and collected microscopy images. B.D.R., I.M., P.V.D.W., and L.A.J. wrote the 883 manuscript, and all authors contributed to its improvement.

884

\section{Competing Interests}

886 The authors declare no competing interests.

887

\section{Data availability}

889 The data sets generated during and/or analysed during the current study are available from the 890 corresponding authors on reasonable request.

\section{References}

898 1. Minami, Y.; Hohfeld, J.; Ohtsuka, K.; Hartl, F. U., Regulation of the heat-shock protein 70 899 reaction cycle by the mammalian DnaJ homolog, Hsp40. J Biol Chem 1996, 271 (32), 19617-24. 
$900 \quad 2 . \quad$ Wall, D.; Zylicz, M.; Georgopoulos, C., The NH2-terminal 108 amino acids of the 901 Escherichia coli DnaJ protein stimulate the ATPase activity of DnaK and are sufficient for lambda 902 replication. J Biol Chem 1994, 269 (7), 5446-51.

903 3. Wall, D.; Zylicz, M.; Georgopoulos, C., The conserved G/F motif of the DnaJ chaperone is 904 necessary for the activation of the substrate binding properties of the DnaK chaperone. J Biol

905 Chem 1995, 270 (5), 2139-44.

906 4. Hartl, F. U., Molecular chaperones in cellular protein folding. Nature 1996, 381 (6583),

907 571-9.

908 5. Cheetham, M. E.; Caplan, A. J., Structure, function and evolution of DnaJ: conservation 909 and adaptation of chaperone function. Cell Stress Chaperones 1998, 3 (1), 28-36.

910 6. Muchowski, P. J.; Wacker, J. L., Modulation of neurodegeneration by molecular 911 chaperones. Nat Rev Neurosci 2005, 6 (1), 11-22.

912 7. Kampinga, H. H.; Craig, E. A., The HSP70 chaperone machinery: J proteins as drivers of 913 functional specificity. Nat Rev Mol Cell Biol 2010, 11 (8), 579-92.

$914 \quad$ 8. Ahmad, A.; Bhattacharya, A.; McDonald, R. A.; Cordes, M.; Ellington, B.; Bertelsen, E. B.; 915 Zuiderweg, E. R. P., Heat shock protein $70 \mathrm{kDa}$ chaperone/DnaJ cochaperone complex employs 916 an unusual dynamic interface. Proc Natl Acad Sci U S A 2011, 108, 18966-18971.

917 9. Flaherty, K. M.; DeLuca-Flaherty, C.; McKay, D. B., Three-dimensional structure of the 918 ATPase fragment of a 70K heat-shock cognate protein. Nature 1990, 346 (6285), 623-8.

919 10. Kityk, R.; Kopp, J.; Mayer, M. P., Molecular Mechanism of J-Domain-Triggered ATP 920 Hydrolysis by Hsp70 Chaperones. Mol Cell 2018, 69 (2), 227-237 e4.

921 11. Rampelt, H.; Kirstein-Miles, J.; Nillegoda, N. B.; Chi, K.; Scholz, S. R.; Morimoto, R. I.; 922 Bukau, B., Metazoan Hsp70 machines use Hsp110 to power protein disaggregation. EMBO J 923 2012, $31(21), 4221-35$.

924 12. Wagner, I.; Arlt, H.; van Dyck, L.; Langer, T.; Neupert, W., Molecular chaperones 925 cooperate with PIM1 protease in the degradation of misfolded proteins in mitochondria. EMBO 926 J 1994, 13 (21), 5135-45.

927 13. Nakatsukasa, K.; Huyer, G.; Michaelis, S.; Brodsky, J. L., Dissecting the ER-associated 928 degradation of a misfolded polytopic membrane protein. Cell 2008, 132 (1), 101-12.

929 14. Nillegoda, N. B.; Stank, A.; Malinverni, D.; Alberts, N.; Szlachcic, A.; Barducci, A.; De Los 930 Rios, P.; Wade, R. C.; Bukau, B., Evolution of an intricate J-protein network driving protein 931 disaggregation in eukaryotes. Elife 2017, 6.

932 15. Jiang, Y.; Possi, P.; Kalodimos, C. G., Structural basis for client recognition and activity of 933 Hsp40 chaperones. Science 2019, 365 (6459), 1313-1319.

934 16. Hageman, J.; Rujano, M. A.; van Waarde, M. A.; Kakkar, V.; Dirks, R. P.; Govorukhina, N.; 935 Oosterveld-Hut, H. M.; Lubsen, N. H.; Kampinga, H. H., A DNAJB chaperone subfamily with 936 HDAC-dependent activities suppresses toxic protein aggregation. Mol Cell 2010, 37 (3), 355-69.

937 17. Karamanos, T. K.; Tugarinov, V.; Clore, G. M., Unraveling the structure and dynamics of 938 the human DNAJB6b chaperone by NMR reveals insights into Hsp40-mediated proteostasis. 939 Proc Natl Acad Sci U S A 2019.

940 18. Månsson, C.; Kakkar, V.; Monsellier, E.; Sourigues, Y.; Härmark, J.; Kampinga, H. H.; 941 Melki, R.; Emanuelsson, C., DNAJB6 is a peptide-binding chaperone which can suppress amyloid 
942 fibrillation of polyglutamine peptides at substoichiometric molar ratios. Cell Stress and 943 Chaperones 2014, 19, 227-239.

944 19. Söderberg, C. A. G.; Månsson, C.; Bernfur, K.; Rutsdottir, G.; Härmark, J.; Rajan, S.; AlKaradaghi, S.; Rasmussen, M.; Höjrup, P.; Hebert, H.; Emanuelsson, C., Structural modelling of the DnaJB6 oligomeric chaperone shows a peptide-binding cleft lined with conserved S/Tresidues at the dimer interface. Scientific Reports 2018, 8 (5199). R.; van Veen, H.; Overkleeft, H.; Goedhart, J.; Kampinga, H. H.; Reits, E. A., The DNAJB6 and DNAJB8 protein chaperones prevent intracellular aggregation of polyglutamine peptides. $J$ Biol Chem 2013, 288 (24), 17225-37.

953 21. Kakkar, V.; Mansson, C.; de Mattos, E. P.; Bergink, S.; van der Zwaag, M.; van Waarde, M. A.; Kloosterhuis, N. J.; Melki, R.; van Cruchten, R. T.; Al-Karadaghi, S.; Arosio, P.; Dobson, C. M.; Knowles, T. P.; Bates, G. P.; van Deursen, J. M.; Linse, S.; van de Sluis, B.; Emanuelsson, C.; Kampinga, H. H., The S/T-Rich Motif in the DNAJB6 Chaperone Delays Polyglutamine Aggregation and the Onset of Disease in a Mouse Model. Mol Cell 2016. 22. Shahmoradian, S. H.; Galez-Montoya, J. G.; Schmid, M. F.; Cong, Y.; Ma, B.; Spiess, C.; Frydman, J.; Ludtke, S. J.; Chiu, W., TRiC's tricks inhibit huntingtin aggregation. eLife 2013, 2 (e00710). 23. Kirchhofer, A.; Helma, J.; Schmidthals, K.; Frauer, C.; Cui, S.; Karcher, A.; Pellis, M.; Muyldermans, S.; Casas-Delucchi, C. S.; Cardoso, M.; Leonhardt, H.; Hopfner, K. P.; Rothbauer, U., Modulation of protein properties in living cells using nanobodies. Nat. Struc. and Mol. Bio. 2010, 17 (1), 133-138.

964 24. Kubala, M. H.; Kovtun, O.; Alexandrov, K.; Collins, B. M., Structural and thermodynamic analysis of the GFP:GFP-nanobody complex. Protein Sci. 2010, 19 (12), 2389-2401. 25. Leitner, A.; Faini, M.; Stengel, F.; Aebersold, R., Crosslinking and Mass Spectometry: An Integrated Technology to Understand the Structure and Function of Molecular Machines. Cell Press 2016, 41 (1), 20-32.

969 26. Leitner, A.; Joachimiak, L. A.; Unverdorben, P.; Walzthoeni, T.; Frydman, J.; Förster, F.; Aebersold, R., Chemical cross-linking/mass spectometry targeting acidic residues in proteins and protein complexes. Proc Natl Acad Sci U S A 2014, 111 (26), 9455-9460.

972 27. Walzthoeni, T.; Joachimiak, L. A.; Rosenberger, G.; Röst, H. L.; Malmström, L.; Leitner, A.;

973 Frydman, J.; Aebersold, R., xTract: software for characterizing conformational changes of protein complexes by quantitative cross-linking mass spectometry. Nat Methods 2015, 12 (12), 1185-1190.

28. Månsson, C.; Arosio, P.; Hussein, R.; Kampinga, H. H.; Hashem, R. M.; Boelens, W. C.; Dobson, C. M.; Knowles, T. P.; Linse, S.; Emanuelsson, C., Interaction of the molecular chaperone DNAJB6 with growing amyloid-beta 42 (Abeta42) aggregates leads to substoichiometric inhibition of amyloid formation. J Biol Chem 2014, 289 (45), 31066-76. 29. Ovchinnikov, S.; Kamisetty, H.; Baker, D., Robust and accurate prediction of residueresidue interactions across protein interfaces using evolutionary information. Elife 2014, 3. 30. Kamisetty, H.; Ovchinnikov, S.; Baker, D., Assessing the utility of coevolution-based residue-residue contact predictions in a sequence- and structure-rich era. Proc Natl Acad Sci U S A 2013, 110 (39), 15674-15679. 
31. Balakrishnan, S.; Kamisetty, H.; Carbonell, J. G.; Lee, S.; Langmead, C. J., Learning generative models for protein fold families. Proteins: Structure, Function, and Bioinformatics 2011, 79 (4), 1061-1078.

32. Marsh, J. A.; Forman-Kay, J. D., Sequence determinants of compaction in intrinsically disordered proteins. Biophys. J. 2010, 98, 2383-2390. 33. Matlahov, I.; van der Wel, P. C. A., Hidden motions and motion-induced invisibility: Dynamics-based spectral editing in solid-state NMR. Methods 2018, 148, 123-135.

992

993 34. Morris, G. A.; Freeman, R., Enhancement of Nuclear Magnetic-Resonance Signals by Polarization Transfer. J Am Chem Soc 1979, 101 (3), 760-762. 35. Lin, H. K.; Boatz, J. C.; Krabbendam, I. E.; Kodali, R.; Hou, Z. P.; Wetzel, R.; Dolga, A. M.; Poirier, M. A.; van der Wel, P. C. A., Fibril polymorphism affects immobilized non-amyloid flanking domains of huntingtin exon1 rather than its polyglutamine core. Nat Commun 2017, 8. 36. Andronesi, O. C.; Becker, S.; Seidel, K.; Heise, H.; Young, H. S.; Baldus, M., Determination of Membrane Protein Structure and Dynamics by Magic-Angle-Spinning Solid-State NMR Spectroscopy. J. Am. Chem. Soc. 2005, 127 (37), 12965-12974. multidimensional chemical shift ranges in proteins from a PACSY database purged using intrinsic quality criteria. J Biomol NMR 2016, 64 (2), 115-30. databases for protein solid-state NMR: $2 \mathrm{D}$ chemical shift maps and amino-acid assignment with secondary-structure information. J Biomol NMR 2013, 56 (2), 155-67. 39. Shen, Y.; Bax, A., SPARTA+: a modest improvement in empirical NMR chemical shift prediction by means of an artificial neural network. J Biomol NMR 2010, 48 (1), 13-22. 40. Mandal, A.; Hoop, C. L.; DeLucia, M.; Kodali, R.; Kagan, V. E.; Ahn, J.; van der Wel, P. C. A., Structural Changes and Proapoptotic Peroxidase Activity of Cardiolipin-Bound Mitochondrial Cytochrome c. Biophys. J. 2015, 109, 1873-1884.

1012 41. Qi, R.; Sarbeng, E. B.; Liu, Q.; Le, K. Q.; Xu, X.; Xu, H.; Yang, J.; Wong, J. L.; Vorvis, C.; Hendrickson, W. A.; Zhou, L.; Liu, Q., Allosteric opening of the polypeptide-binding site when an Hsp70 binds ATP. Nat. Struct. Mol. Biol. 2013, 20, 900-907. structure of the J domain of DnaJ homolog subfamily B member 8. RIKEN Structural Genomics/Proteomics Initiative (RSGI), 2006.

43. Mayer, M. P.; Laufen, T.; Paal, K.; McCarty, J. S.; Bukau, B., Investigation of the interaction between DnaK and DnaJ by surface plasmon resonance spectroscopy. $\mathrm{J} \mathrm{Mol} \mathrm{Biol}$ 1999, 289 (4), 1131-1134.

44. Martin, E. W.; Holehouse, A. S.; Peran, I.; Farag, M.; Incicco, J. J.; Bremer, A.; Grace, C. R.; Sorano, A.; Pappu, R. V.; Mittag, T., Valence and Patterning of Aromatic Residues Determine the Phase Behavior of Prion-Like Domains. Science 2020, 367 (6478), 694-699.

45. Holehouse, A. S.; Ahad, J.; Das, R. K.; Pappu, R. V., CIDER: Classification of Intrinsically Disordered Ensemble Regions. Biophys. J. 2015, 108, 228 a. 
1027 I., Distinct tau prion strains propagate in cells and mice and define different tauopathies.

1028 Neuron 2014, 82 (6), 1271-1288.

1029 47. Leitner, A.; Walzthoeni, T.; Aebersold, R., Lysine-specific chemical cross-linking of protein complexes and identification of cross-linking sites using LC-MS/MS and the xQuest/xProphet software pipeline. Nat. Protocols 2014, 9, 120-137. Doan, M.; Ding, L.; Rafelski, S. M.; Thirstrup, D.; Wiegraebe, W.; Singh, S.; Becker, T.; Caicedo, J. C.; Carpenter, A. E., CellProfiler 3.0: Next-generation image processing for biology. PLoS Biol. 2018, 16 (7), e2005970.

49. Schneider, C. A.; Rasband, W. S.; Eliceiri, K. W., NIH Image to ImageJ: 25 years of image analysis. Nat. Methods 2012, 9, 671-675. properties of rigid proteins from atomic and residue-level models. Biophys. J. 2011, 101, 892898.

51. Altschul, S. F.; Gish, W.; Miller, W.; Myers, E. W.; Lipman, D. J., Basic Local Alignment Search Tool. J. Mol. Bio. 1990, 215, 403-410. BLAST and PSI-BLAST: a new generation of protein database search programs. Nucleic Acids 1045 Research 1997, 25, 3389-3402. 53. Madeira, F.; Park, Y. M.; Lee, J.; Buso, N.; Gur, T.; Madhusoodanan, N.; Basutkar, P.; Tivey, A. R. N.; Potter, S. C.; Finn, R. D.; Lopez, R., The EMBL-EBI search and sequence analysis tools APIs in 2019. Nucleic Acids Research 2019, 47 (W1), 636-641.

54. Pei, J.; Grishin, N. V., AL2CO: calculation of positional conservation in a protein sequence alignment. Bioinformatics 2001, 17 (8), 700-712. devices for routine sample preparation in biomolecular magic-angle-spinning NMR. J Biomol NMR 2017, 67 (3), 165-178. Decoupling in Rotating Solids. J Chem Phys 1995, 103 (16), 6951-6958. CA, 2004. multidimensional spectral processing system based on UNIX pipes. Journal of Biomolecular NMR 1995, 6, 277-293. biomolecular NMR spectroscopy. Bioinformatics 2015, 31 (8), 1325-1327. FL, 2006. 
1069 62. Schuck, P., Size-distribution Analysis of Macromolecules by Sedimentation Veelocity 1070 Ultracentrifugation and Lamm Equation Modeling. Biophys. J. 2000, 78 (3), 1606-1619.

1071 63. Micsonai, A.; Wien, F.; Bulyáki, E.; Kun, J.; Moussong, E.; Lee, Y.; Goto, Y.; Réfrégiers, M.; 1072 Kardos, J., BeStSel: a web server for accurate protein secondary structure predicition and fold 1073 recognition from the circular dichroism spectra. Nucleic Acids Research 2018, 46 (W), 315-322. 1074 64. Micsonai, A.; Wien, F.; Kernya, L.; Lee, Y.; Goto, Y.; Réfrégiers, M.; Kardos, J., Accurate 1075 scondary structure prediction and fold recognition for circular dichroism spectroscopy. Proc 1076 Natl Acad Sci U S A 2015.

1077 65. Scheuermann, T. H.; Padrick, S. B.; Gardner, K. H.; Brautigam, C. A., On the acquisition 1078 and analysis of microscale thermophoresis data. Anal Biochem. 2016, 496, 79-93.

1079 66. Eschenfeldt, W. H.; Lucy, S.; Millard, C. S.; Joachimiak, A.; Mark, I. D., A family of LIC 1080 vectors for high-throughput cloning and purification of proteins. Methods Mol. Biol. 2009, 498, $1081 \quad 105-115$.

1082

1083

1084 
a

\begin{tabular}{|l|l|l|l|}
\hline 147 & \multicolumn{3}{c}{$148186 \quad 232$} \\
\hline J-Domain & G/F & S/T & CTD \\
\hline
\end{tabular}

\begin{tabular}{|l|l|l|l|l|}
\hline J-Domain & G/F & S/T & CTD \\
\hline
\end{tabular}

Clover

b

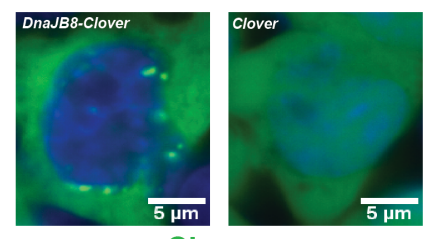

C

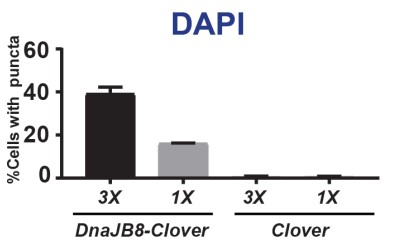

e

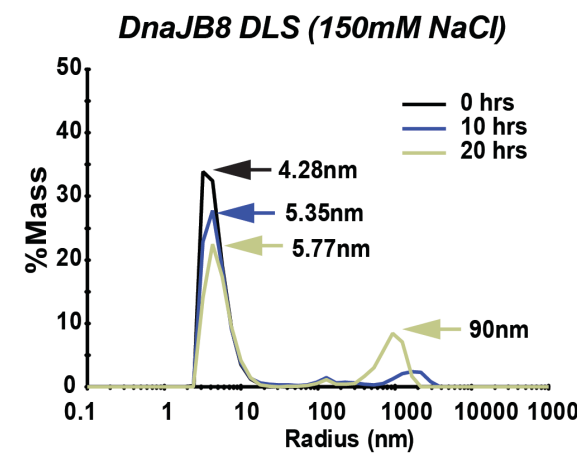

g

DnaJB8 DLS (285mM NaCl)

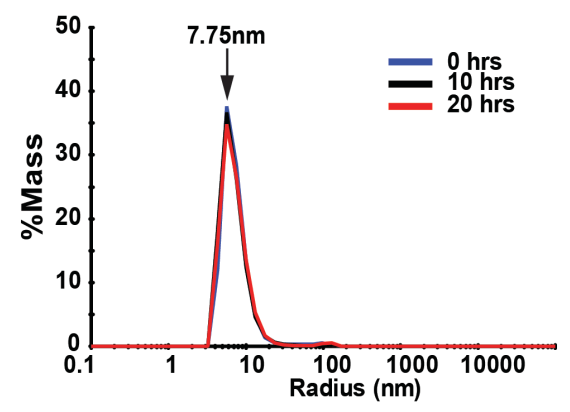

\section{Figure 1}

DnaJB8-Clover

Clover

d

DnaJB8-Clover XL-MS

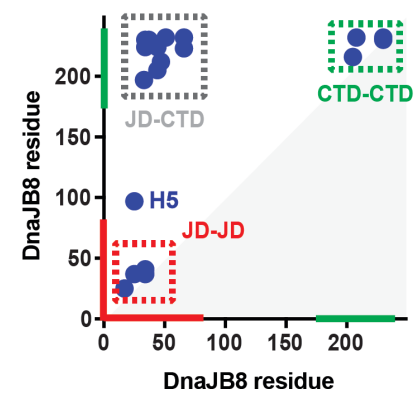

f

DnaJB8 XL-MS (150mM NaCl)

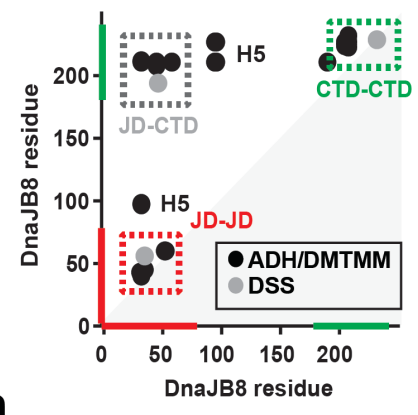

h

DnaJB8 XL-MS (285mM NaCl)

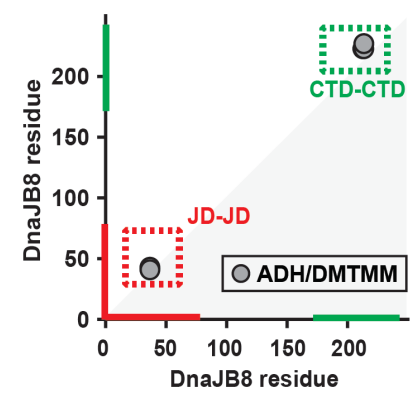

Figure 1. DnaJB8 architecture defined by domain-domain interactions. (a) Domain maps for DnaJB8 used in the in vitro experiments and the DnaJB-Clover and Clover constructs used in the mammalian cell experiments. DnaJB8 colored according to domain annotation: JD (red), G/F rich (blue), S/T rich (cyan), and CTD (green). Clover is colored pale green. (b) Representative images of triplicate populations of 300,000 cells expressing DnaJB8-mClover3(left) and mClover3(right). Clover and DAPI fluorescence signal are shown in green and blue, respectively. $5 \mu \mathrm{m}$ scale bar is shown in white. (c) Quantification of DnaJB8-Clover and Clover puncta in high (3X) and low (1X) 
protein level expressing cell lines. In each analysis at least 2,000 cells were counted by the CellProfiler software. Puncta were manually counted by two independent observers, with data reported as averages with standard deviation. (d) XL-MS contact map of DnaJB8-Clover crosslinks identified using DMTMM and ADH. The axes are colored in red and green for JD and CTD, respectively. Crosslink pairs between JD-CTD, JD-JD and CTD-CTD are shown in dashed boxes colored grey, red and green, respectively. Contacts to helix 5 are denoted with H5. (e) Histogram of overall $R_{h}$ of DnaJB8 in 1xPBS 150mM NaCl from DLS at times Ohrs (black), 10hrs (blue), and $20 \mathrm{hrs}$ (gold) with arrows indicating $R_{\mathrm{h}}$ peaks for each time point. Over time, there was a depletion in particle sizes $<10 \mathrm{~nm}$ and an increase in particles $\sim 100-1000 \mathrm{~nm}$. (f) XL-MS contact map of DnaJB8 crosslinks identified using DMTMM and ADH (black) and DSS (grey). The axes are colored in red and green for JD and CTD, respectively. Crosslink pairs between JD-CTD, JDJD and CTD-CTD are shown in a dashed box colored grey, red and green, respectively. Contacts to helix 5 are denoted with $\mathrm{H} 5$. (g) Histogram of overall $\mathrm{R}_{\mathrm{h}}$ of DnaJB8 in $1 \mathrm{xPBS} 285 \mathrm{mM} \mathrm{NaCl}$ at times $0 \mathrm{~h}$ (blue), $10 \mathrm{~h}$ (black), and $20 \mathrm{~h}$ (red) with arrows indicating $\mathrm{R}_{\mathrm{h}}$ peaks for each time point. Over time, there is no change in the species of particle sizes $<10 \mathrm{~nm}$ and no appearance of particles $\sim 100-1000 \mathrm{~nm}$. (h) Contact map of DnaJB8 crosslinks identified using ADH/DMTMM in the presence of $285 \mathrm{mM} \mathrm{NaCl}$. The axes are colored in red and green for JD and CTD, respectively. JD-JD and CTD-CTD crosslinks are shown in dashed boxes colored in red and

1112 green, respectively.

1113

1114

1115

1116

1117

1118

1119

1120

1121

1122

1123

1124

1125

1126

1127

1128

1129

1130

1131

1132

1133

1134

1135 
a
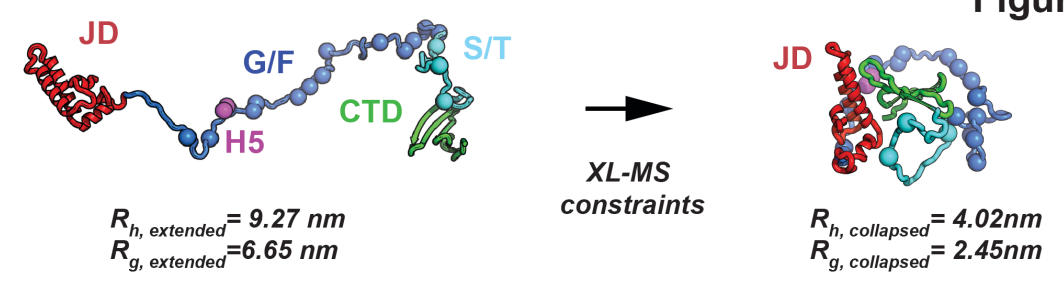

b
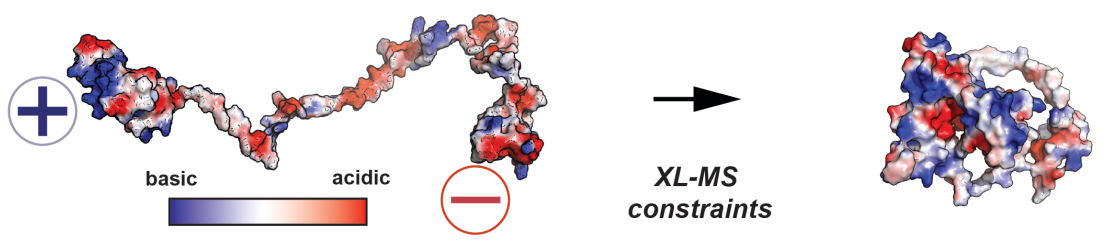

C

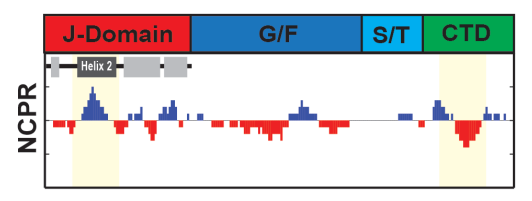

e
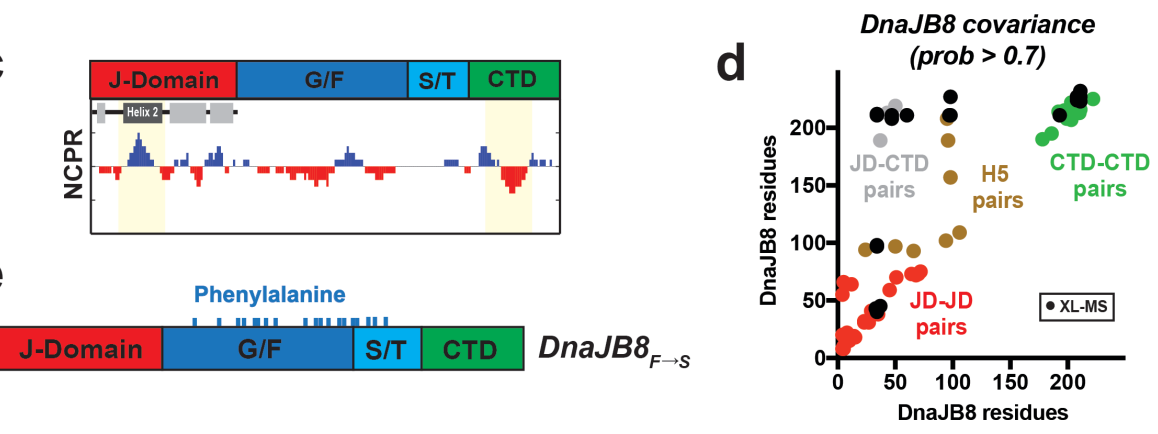

f

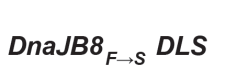

g

$D N a J B 8_{F \rightarrow S} S E C$ MALS

h
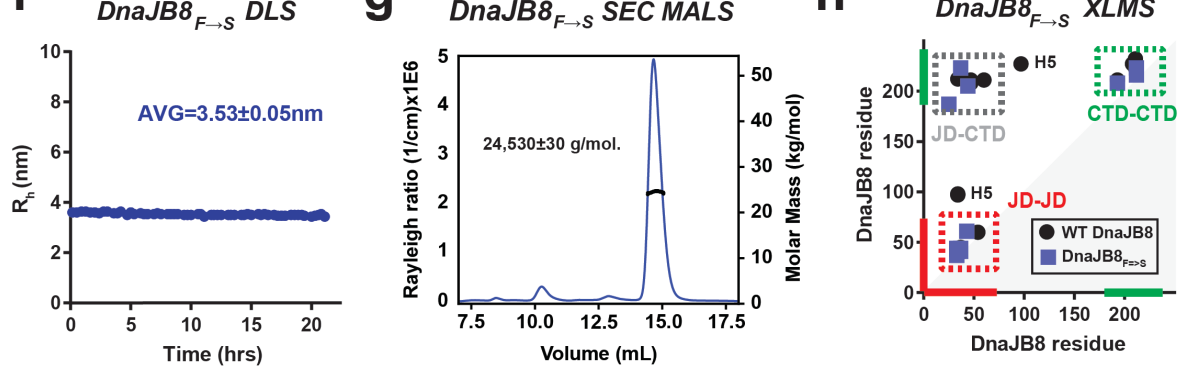

i
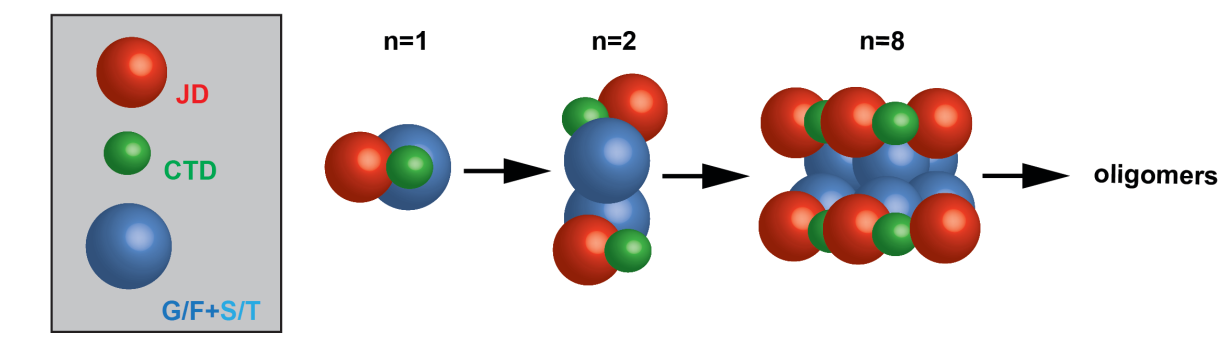

Figure 2. Model for the JD-CTD contacts in a DnaJB8 monomer. (a) XL-MS-based refinement of full length expanded DnaJB8 monomer. Cartoon representation of DnaJB8 in fully expanded conformation (left) and collapsed conformation (right), colored by domain as in Fig. 1. Aromatic amino acids in the G/F and S/T domains are shown as spheres and colored according to the domain. Residues in helix 5 (H5) are shown as magenta spheres. Collapsed conformation model was selected from 1,000 Rosetta ab initio generated models using a relax protocol. $R_{g}$ and $R_{h}$ values were calculated from the structural model in Rosetta and HYDROPRO, respectively. (b) 
1145 Charge complementary surfaces on the JD and CTD mediate the interaction. Highly acidic potential is shown in red (- sign) and highly basic in blue (+ sign). (c) Net charge per residue (NCPR) distribution, defined as the average charge over a 10-residue window, highlights charge complementarity between basic and acidic residues on the JD and CTD, respectively (coloring as in Fig. 1). (d) GREMLIN sequence-based covariance analysis identified high confidence covarying amino acids on DnaJB8 that localize within the JD (red), within CTD (green), with H5 (brown) and across JD-CTD (grey). XL-MS links for full length DnaJB8 (black dots) overlap with the covarying regions. Covarying positions localizing to amino acids in G/F domain are shown in brown and co-localize with XL-MS crosslinks. (e) Domain map of the DnaJB8 $\mathrm{F}_{\mathrm{F}} \mathrm{S}$ mutant, with mutated phenylalanine positions marked by cyan ticks. (f) DLS time course of the DnaJB8 ${ }_{F \rightarrow S}$ mutant. The average $R_{h}$ was calculated to be $3.53 \pm 0.05 \mathrm{nnm}$. $(\mathrm{g})$ SEC-MALS of CTD $170-232$ shows a single peak that was calculated to have a molar mass of $24,530 \pm 30 \mathrm{~g} / \mathrm{mol}$ consistent with a monomer. (h) XL-MS contact map showing ADH/DMTMM crosslinks for WT DnaJB8 and DnaJB8 ${ }_{F \rightarrow S}$ mutant. The axes are colored in red and green for JD and CTD, respectively. Crosslink pairs between JD-CTD, JD-JD and CTD-CTD are shown in a dashed box colored grey, red and green, respectively. Contacts to helix 5 in WT DnaJB8 are denoted with H5. (i) Schematic of DnaJB8 species observed in solution based on DLS dimensions. Domains are shown as JD (red spheres), CTD (green spheres), and G/F+S/T (light blue spheres). The average $R_{h}$ of DnaJB8 $8_{F \rightarrow S}$ $(3.53 \pm 0.05 \mathrm{nnm})$ and DnaJB8 ab initio Rosetta model $(4.02 \mathrm{~nm})$ are assigned to the monomer. The $R_{h}$ of WT DnaJB8 begins as a $4.28 \mathrm{~nm}$ species and grows to $5.77 \mathrm{~nm}$ over $20 \mathrm{hrs}$. Size and volume estimates from the structural models suggest DnaJB8 exists as small species ranging from a monomer to octamer likely dominated by a dimer and over time maturing into large oligomers. 
Figure 3

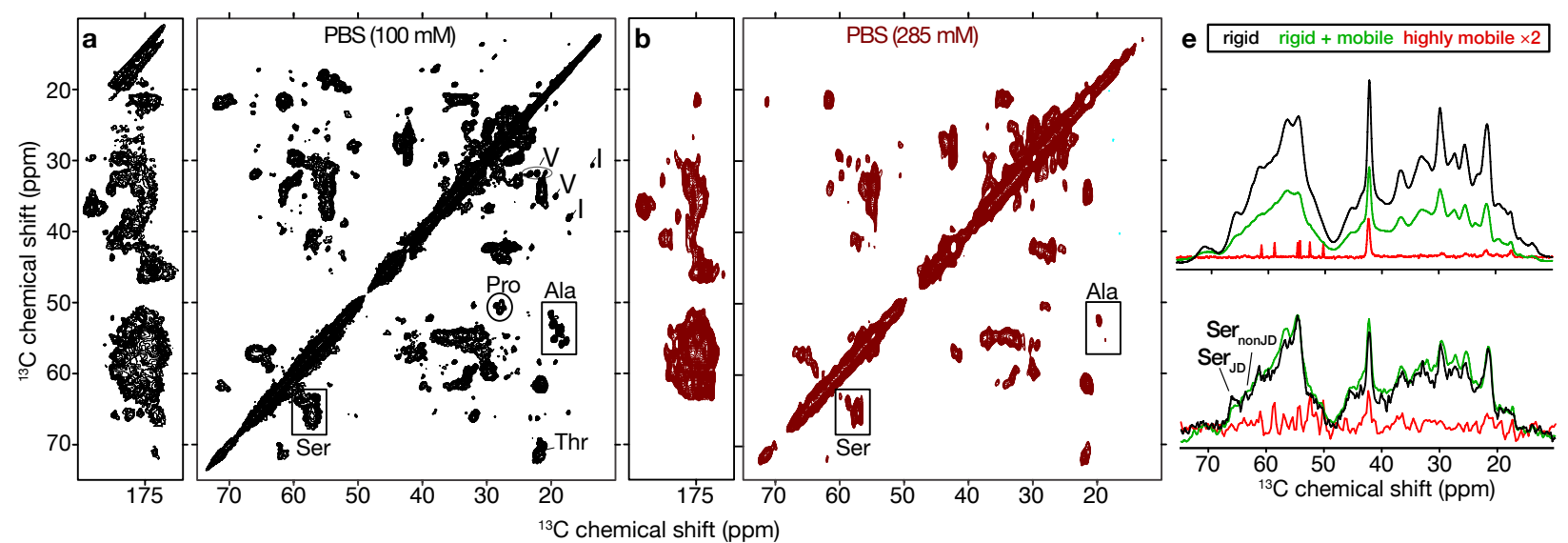

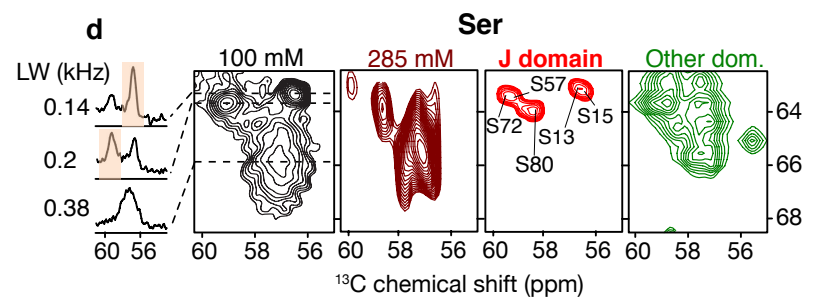

Figure 3. Solid-state NMR of DnaJB8 oligomers at physiological and high ionic strength. (a) $2 \mathrm{D}{ }^{13} \mathrm{C}-{ }^{13} \mathrm{C}$ ssNMR spectrum of $\mathrm{U}-{ }^{13} \mathrm{C},{ }^{15} \mathrm{~N}$-labeled DnaJB8 oligomers in PBS (100 mM NaCl), using 25 ms DARR mixing. (b) Corresponding 2D ssNMR spectrum in PBS with $285 \mathrm{mM} \mathrm{NaCl}$. (c-d) Boxed Ala and Ser regions from panels "a" and "b". In PBS, the experimental Ala and Ser peak patterns (black) are well resolved and similar to those expected for folded JD in solution (red). At elevated ionic strength (brown) these narrow peaks are missing. Green spectra (right) represent simulated signals predicted for our models of the non-JD domains, shown with enhanced broadening reflecting the heterogeneity seen in the experiments. 1D spectra on far left show slices through the experimental 2D data, with selected peak widths (in $\mathrm{kHz}$ ). (e) ${ }^{13} \mathrm{C}$ 1D spectra, in PBS (top) and with $285 \mathrm{mM} \mathrm{NaCl}$ (bottom), that show rigid residues (black, CP), rigid and mobile residues (green, SPE) and only mobile residues (red, INEPT). See also text and Supplementary Fig. 3. 
a

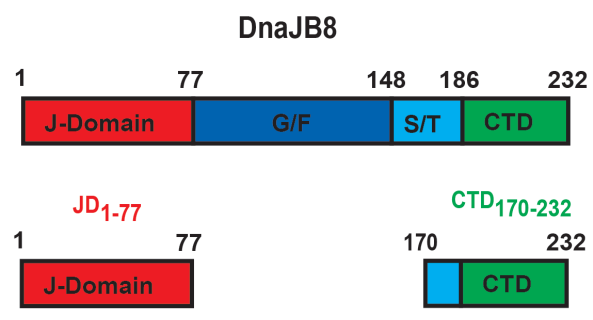

C

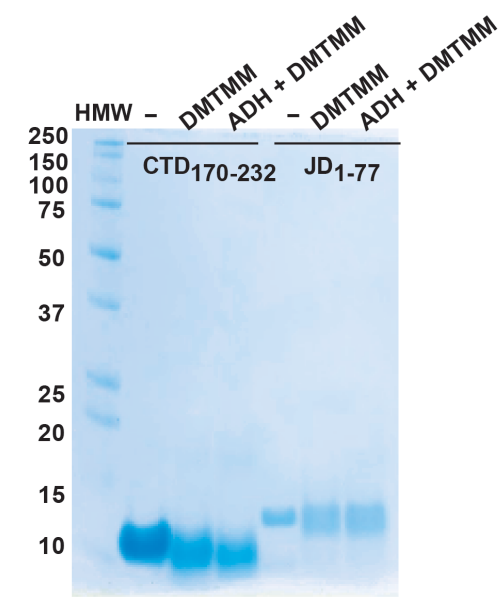

e

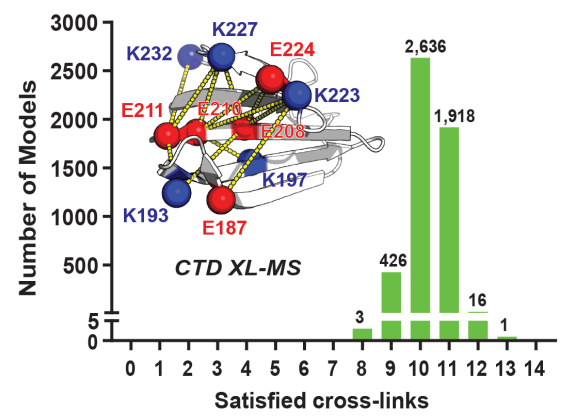

b

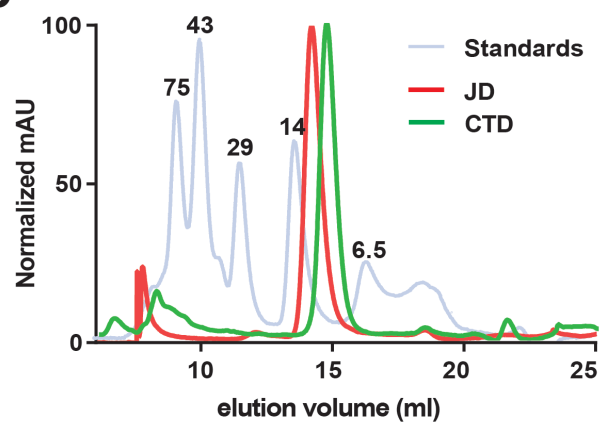

d

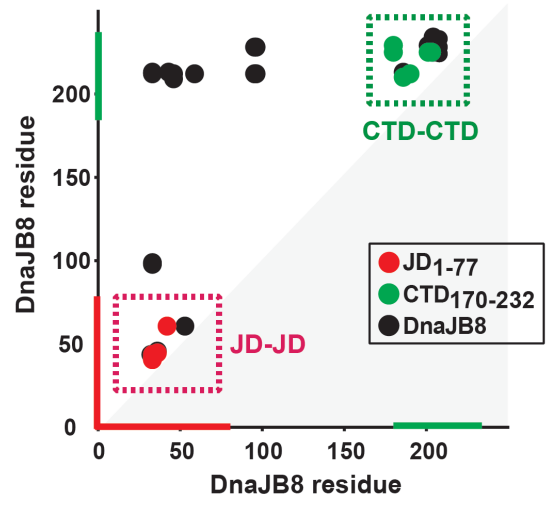

f

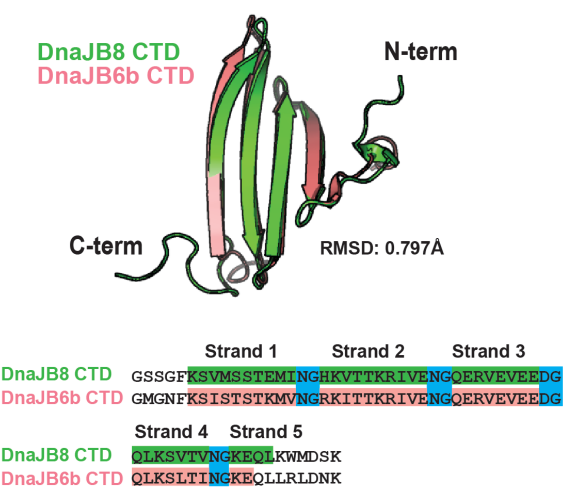

Figure 4. Isolated JD and CTD proteins are monomeric. (a) Cartoon schematic for the full length DnaJB8 and domain fragments $\mathrm{JD}_{1-77}$ and $\mathrm{CTD}_{170-232 .}$. (b) Representative SEC profiles of $\mathrm{JD}_{1-77}(\mathrm{red}), \mathrm{CTD}_{170-232}$ (green) and LMW standards (blue). $\mathrm{JD}_{1-77}$ and CTD ${ }_{170-232}$ elute at apparent molecular weights of $14 \mathrm{kDa}$ and $6.5 \mathrm{kDa}$, respectively. (c) SDS-PAGE coomassie gel of crosslinked $\mathrm{JD}_{1-77}$ and $C T \mathrm{D}_{170-232}$ reacted with either DMTMM only or DMTMM with ADH. (d) Contact map of ADH/DMTMM crosslinks identified for $\mathrm{JD}_{1-77}(\mathrm{red})$, CTD ${ }_{170-232}$ (green) and full length DnaJB8 (black). The axes are colored in red and green for JD and CTD, respectively. Crosslink pairs between JD-JD and CTD-CTD are shown in dashed boxes colored in red and green, respectively. (e) Histogram of the number of intra-domain crosslinks that are consistent with crosslink chemistry geometry ("satisfied") in the ensemble of 5000 models. One model satisfies 13 out of 14 possible crosslinks identified in our experiments. Crosslinks are mapped onto best matching CTD structural model (inset), shown in white cartoon representation. Sites of crosslink are shown as red or blue spheres, for D/E and K, respectively. Dashed yellow lines connect linked 
1211 amino acid pairs. (f) Overlay of our DnaJB8 CTD model generated by ab initio ROSETTA (green) 1212 with the published DnaJB6b $\triangle S T$ CTD (salmon) (PDB:6U3R). The CTD sequences of DNAJB8 1213 and DNAJB6 are shown with each $\beta$-strand highlighted and conserved NG and DG turns in blue.

1214

1215

1216

1217

1218

1219

1220

1221

1222

1223

1224

1225

1226

1227

1228

1229

1230

1231

1232

1233

1234

1235

1236

1237

1238

1239

1240

1241

1242

1243

1244

1245

1246

1247 
a
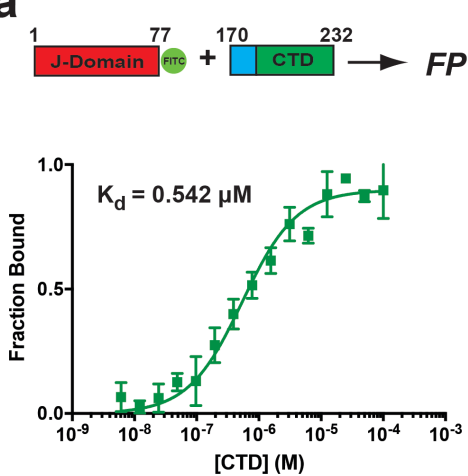

b
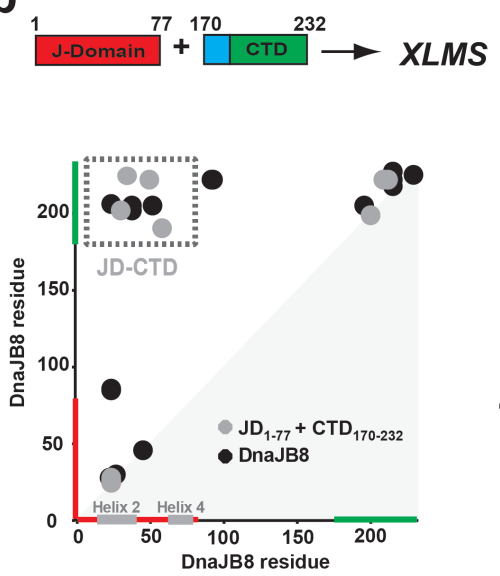

C

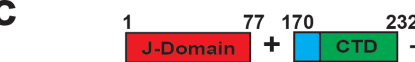

232

Chemical Shifts

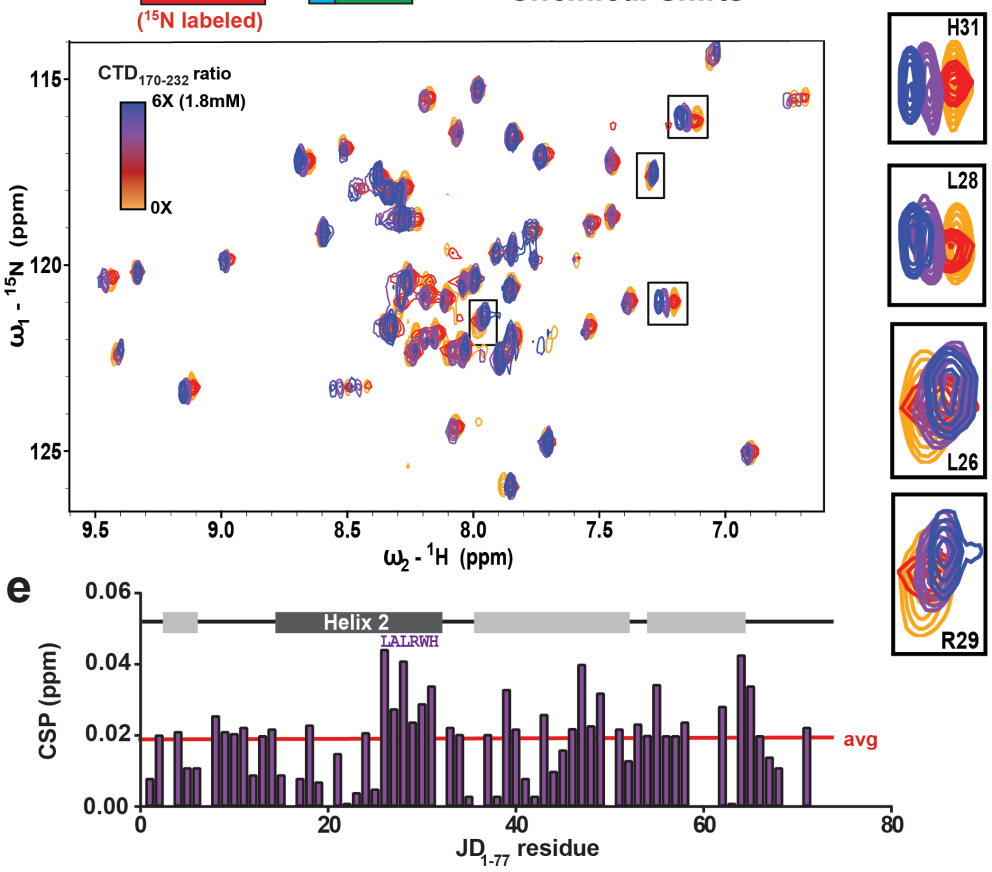

f

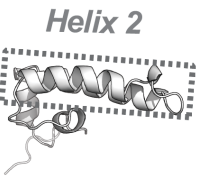

d Figure 5
1248

1249

1250

1251

1252

1253

1254

1255

1256

1257

1258

1259

1260

1261

1262

1263

1264

1265

1266

1267

1268

1269

1270

Figure 5. JD and CTD interact through charge complementary surfaces. (a) Schematic of the JD $_{1-77}$-FITC (FITC dye is shown as a green circle) and CTD ${ }_{170-232}$ constructs used in fluorescence polarization (FP) experiments. FP titration measuring interaction between $\mathrm{JD}_{1-77^{-}}$ FITC and a concentration range of unlabeled CTD $_{170-232}$. FP experiments were performed in triplicate and shown as averages with standard deviation. (b) Schematic of the JD 1-77 $_{\text {and CTD }}$ an232 constructs used in the XL-MS experiments. Contact map of ADH/DMTMM crosslinks identified from an incubated $\mathrm{JD}_{1-77}$ and $\mathrm{CTD}_{170-232}$ sample (grey) and full length DnaJB8 (black). The axes are colored in red and green for JD and CTD, respectively. Helix 2 and 3 are shown in grey on the x-axis. Crosslink pairs between JD-CTD are shown in a dashed box colored in grey. (c) Schematic for the solution NMR chemical shift experiment with U- ${ }^{15} \mathrm{~N}$ JD titrated with unlabeled CTD. HSQC solution NMR spectrum of $300 \mu \mathrm{M}{ }^{15} \mathrm{~N}$-labelled $\mathrm{JD}_{1-77}$ against a titration of CTD $170-232$ : 0x (yellow), 1x (red), 3x (purple), 6x (blue). DnaJB8 JD peak assignments were transferred from deposited data (BMRB:11417). (d) Insets of peaks with highest observed chemical shifts: H31, L28, L26, R29. Coloring as in c. (e) Histogram of chemical shift perturbations (CSP) from 3x CTD experiment by residue. Average CSP of $\sim 0.019 \mathrm{ppm}$ is denoted by the red line (excludes prolines). The sequence of last 6 residues of helix 2 are marked above their respective peaks. (f) DnaJB8 JD structure illustrating the location of helix 2 (pdbid:2DMX). (g) Mapping CSP values onto the DnaJB8 JD structure, shown in surface representation and colored according to $\Delta \delta$ from low $(0.0$ ppm) in yellow to high (red; $0.04 \mathrm{ppm}$ ). (h) Electrostatic potential mapped onto DnaJB8 JD structure shown in surface representation. Highly acidic potential is shown in red and highly basic in blue. 
a

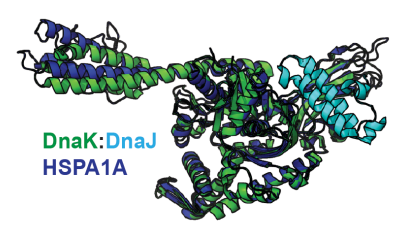

d

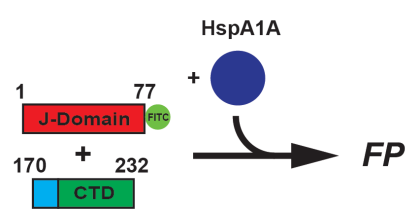

e

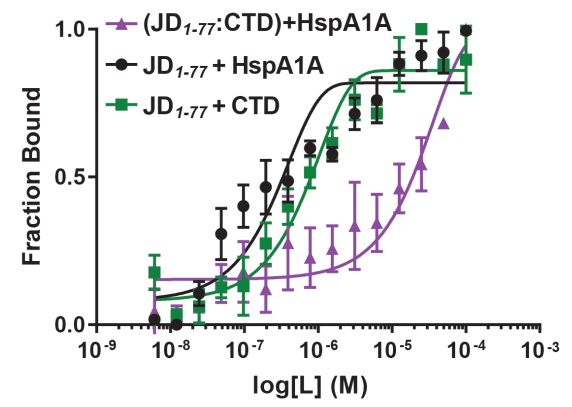

1271

1272

1273

1274

1275

1276

1277

1278

1279

1280

1281

1282

1283

1284

1285

1286

1287

1288

1289

1290

1291

1292 b

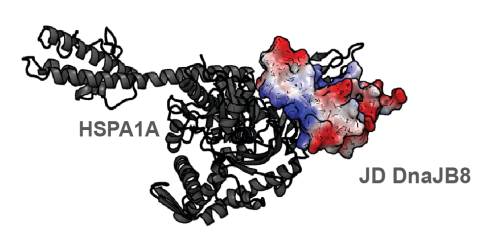

f

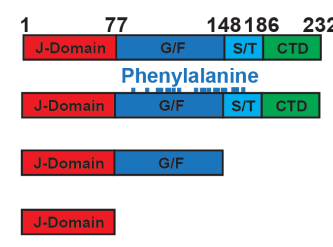

g

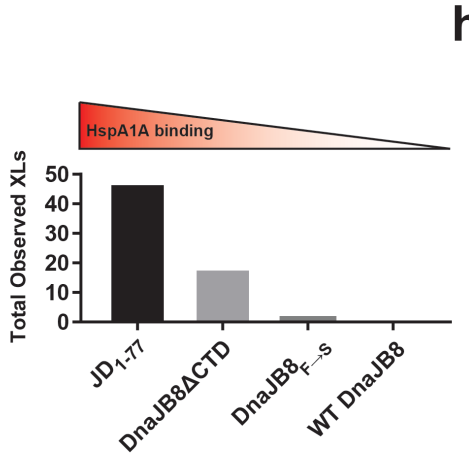

C
Figure 6
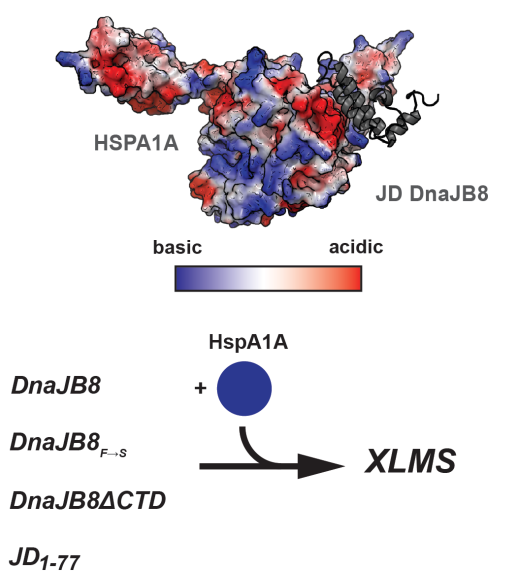

h

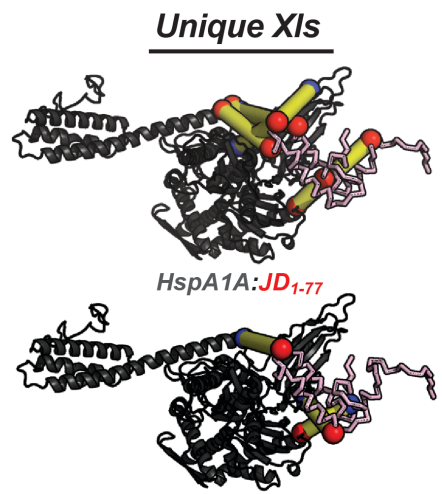

HspA1A:DnaJB8 $\triangle$ CTD

Figure 6. CTD and HSPA1A compete for the same basic binding surface on DnaJB8aD. (a) Structural superposition of a representative HspA1A structural homology model (blue) with a crystal structure of DnaK-DnaJ (green and cyan, respectively; PDBID: 5NRO) shows good agreement. (b-c) Electrostatic surface potential of DnaJB8 JD docked into the JD binding site on HspA1A (shown in black cartoon representation). Basic surface on helix 2 docks onto the HspA1A surface. Electrostatic surface potential of HspA1A with docked DnaJB8 JD in black cartoon representation. The HspA1A surface presents an acidic face that complements the basic DnaJB8 JD surface. Highly acidic potential is shown in red and highly basic is shown in blue. (d) Experimental workflow used to determine competition between Hsp70 and CTD $170-232$ for JD 1-77FITC binding (dye shown as a green circle). (e) Normalized FP binding curves measuring affinity between fluorescent JD and added CTD (green) or added Hsp70 (grey). Preincubation with CTD followed by addition of Hsp70 (purple) shows delay in binding consistent with a competitive binding model. FP experiments were performed in triplicate and shown as averages with standard deviation. (f) XL-MS-based experimental workflow used to determine contribution of CTD to regulate JD binding to Hsp70. WT DnaJB8, DnaJB8 $\Delta$ CTD, DnaJB8 ${ }_{F \rightarrow S}$ and JD 1-77 $_{\text {DnaJB8 }}$ variants were used to form complexes with HspA1A. (g) Summary of total intermolecular crosslinks identified across three XL-MS experiments between the JD and HspA1A for four complexes: JD 1-77:HspA1A, DnaJB8 $\Delta$ CTD:HspA1A, WT DnaJB8:HspA1A and DnaJB8 $_{F \rightarrow s}: H s p A 1 A$. (h) Unique intermolecular crosslinks identified across three datasets in the $J_{1-77}: H s p A 1 A$ and DnaJB8 $\Delta$ CTD:HspA1A complexes mapped onto the JD-HspA1A model. JD 
1293 is shown in pink ribbon representation and HspA1A in black cartoon representation. Sites of 1294 crosslink are shown as red or blue spheres for aspartic/glutamic acid and lysine, respectively. 1295 Yellow lines connect linked amino acid pairs. 


\section{a. Polydisperse DnaJB8 assemblies}

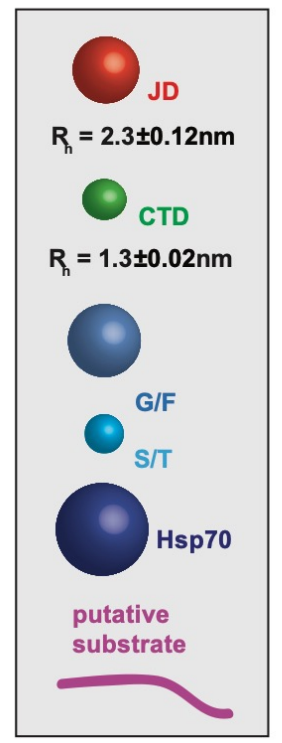

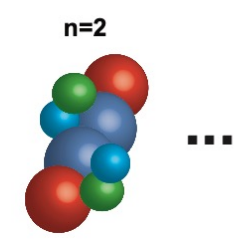

$3.53 \mathrm{~nm}$

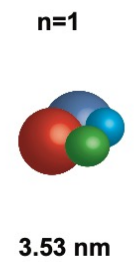

b. Hypothesized impact of substrate binding
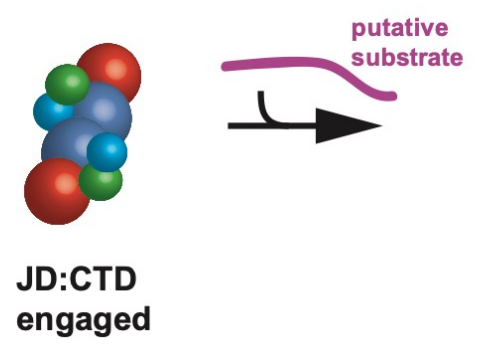
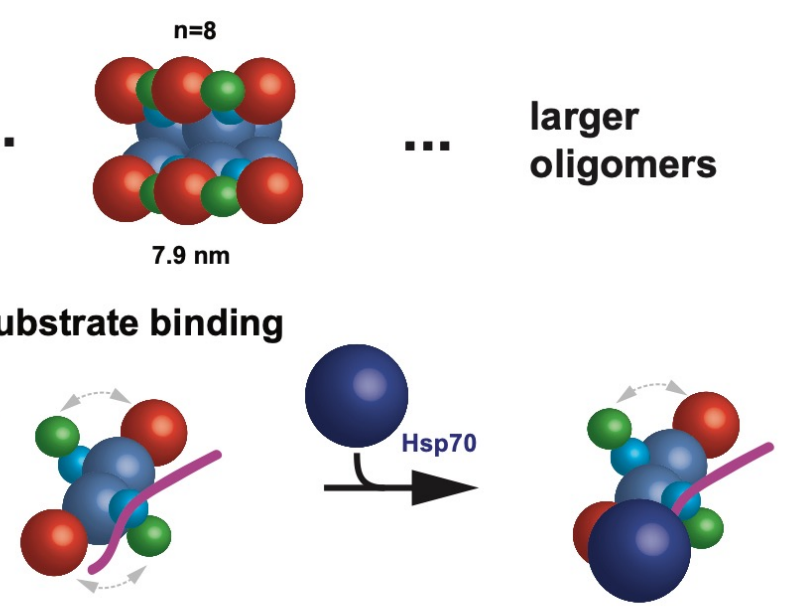

Figure 7. Proposed model for DnaJB8:HspA1A:Substrate relationship

Schematic of proposed DnaJB8 model. Domains are shown as JD (red spheres), CTD (green spheres), G/F (blue spheres), S/T (light blue spheres), and also HspA1A (dark blue spheres) and substrate (purple line) are shown. DnaJB8 domain sizes are displayed scaled to the relative $R_{h}$ values derived from DLS experiments (HspA1A not drawn to scale). (a) DnaJB8 forms a fundamental oligomeric species through aromatic contacts in the $G / F$ and $S / T$ domains ranging from monomer to octamer. (b) The JD:CTD engaged state, where the JD is stabilized by CTD and helix $5(\mathrm{G} / \mathrm{F})$ contacts, can form larger polydisperse oligomers (>100nm). The JD:CTD disengaged state (bottom) is needed to engage with HspA1A. We illustrate our hypothesis where substrate binding may allosterically disrupt the JD:CTD interaction to allow the recruitment of HspA1A to the freed JD-CTD binding face, enabling subsequent handoff of substrate to HspA1A. 
Auto-regulatory J-domain interactions control Hsp70 recruitment to

1364 Bryan Ryder, Irina Matlahov, Sofia Bali, Jaime Vaquer-Alicea, Patrick C.A. van der Wel, Lukasz

A. Joachimiak

1366

1367

1368

1369

1370

1371

1372

1373

1374

1375

1376

1377

1378

1379

1380

Supplementary Data

1382

Supplementary Data 1. Primary XL-MS data for DnaJB8 and its domains. 


\section{Supplemental Tables}

1397

1398 Supplementary Table 1. Residue distribution per domain with net charge for each domain.

\begin{tabular}{|l|l|l|l|l|l|l|l|l|l|l|l|l|l|l|l|l|l|l|l|l|l|l|l|}
\hline $\begin{array}{l}\text { Domain } \\
\text { aa. } \\
\text { length }\end{array}$ & \multirow{2}{*}{$\begin{array}{l}\text { Domai } \\
\mathbf{1 - 7 5}\end{array}$} & $\mathbf{A}$ & $\mathbf{R}$ & $\mathbf{N}$ & $\mathbf{D}$ & $\mathbf{Q}$ & $\mathbf{E}$ & $\mathbf{G}$ & $\mathbf{H}$ & $\mathbf{I}$ & $\mathbf{L}$ & $\mathbf{K}$ & $\mathbf{M}$ & $\mathbf{F}$ & $\mathbf{P}$ & $\mathbf{S}$ & $\mathbf{T}$ & $\mathbf{Y}$ & $\mathbf{V}$ & $\mathbf{W}$ & $\mathbf{C}$ & $\begin{array}{l}\text { for } \\
\text { domain }\end{array}$ \\
\hline & JD & 8 & 5 & 3 & 6 & 1 & 7 & 2 & 1 & 1 & 6 & 0 & 1 & 1 & 3 & 7 & 0 & 5 & 4 & 2 & 1 & +2 \\
\hline $\mathbf{7 6 - 1 4 8}$ & G/F & 6 & 5 & 2 & 5 & 0 & 5 & 3 & 2 & 1 & 2 & 0 & 1 & 4 & 6 & 6 & 3 & 2 & 0 & 1 & 0 & \\
\hline $149-185$ & S/T & 0 & 0 & 1 & 0 & 0 & 1 & 7 & 1 & 0 & 1 & 1 & 3 & 4 & 0 & 5 & 4 & 0 & 1 & 0 & 1 & \\
\hline $\mathbf{1 8 6 - 2 3 2}$ & CTD & 0 & 2 & 3 & 2 & 3 & 6 & 4 & 7 & 2 & 2 & 6 & 1 & 0 & 0 & 2 & 3 & 0 & 6 & 1 & 0 & 0 \\
\hline
\end{tabular}

1399

1400

1401

1402

1403

1404

1405

1406

1407

1408

1409

1410

1411

1412

1413

1414

1415

1416

1417

1418

1419

1420

1421

1422

1423

1424

1425 
a

$$
\frac{\text { DnaJB8-Clover }}{3 \times 1 \times} \frac{\text { Clover }}{3 \times 1 \times}
$$

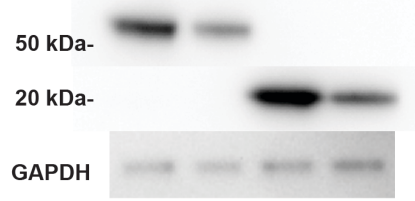

b

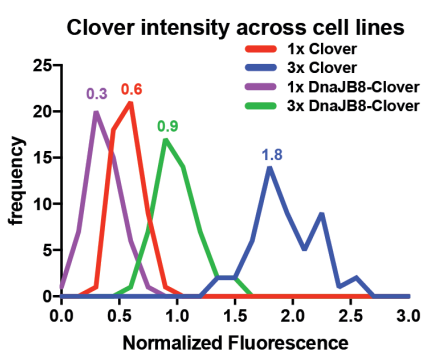

C

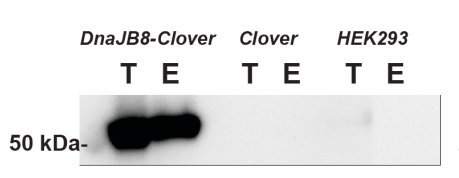

$\alpha$-DnaJB8

JB8-Clover Clover HEK293

$\begin{array}{llllll}\text { T } & E & \text { T } & E & T & \text { E }\end{array}$

\section{Supplementary Figure 1}
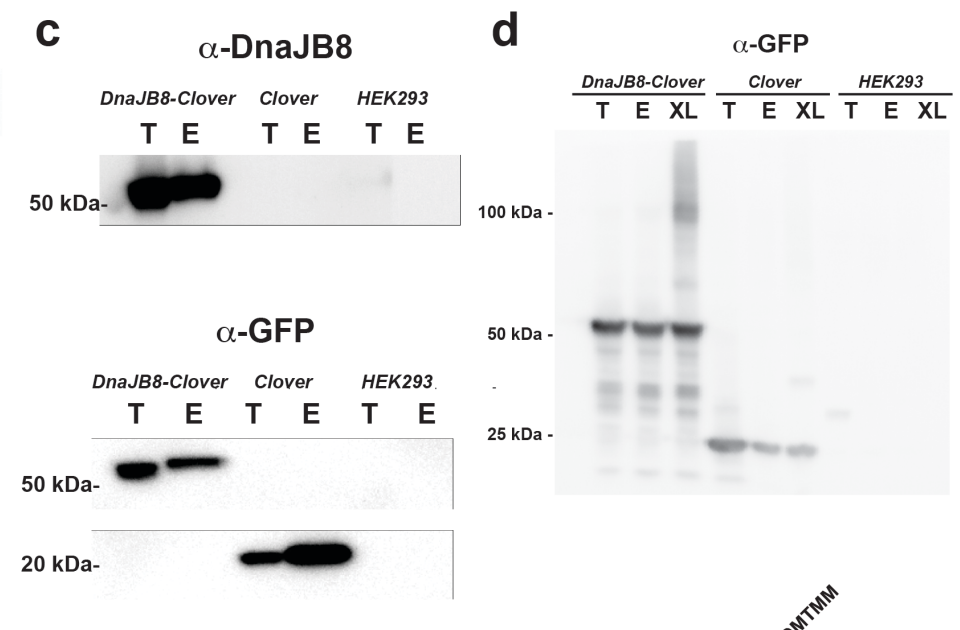

e

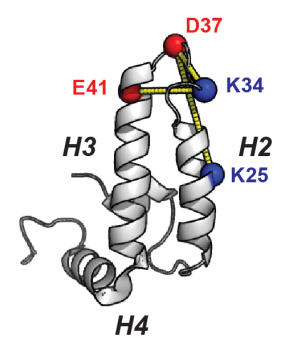

$J D-J D$ ex vivo Contacts

h

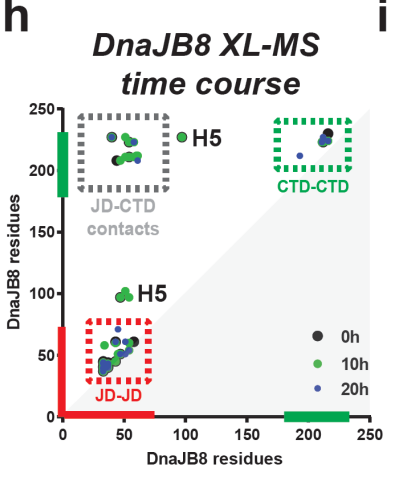

f

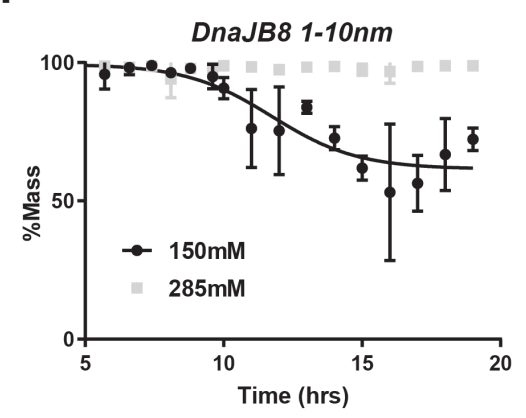

j

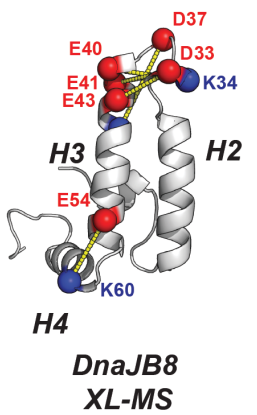

g

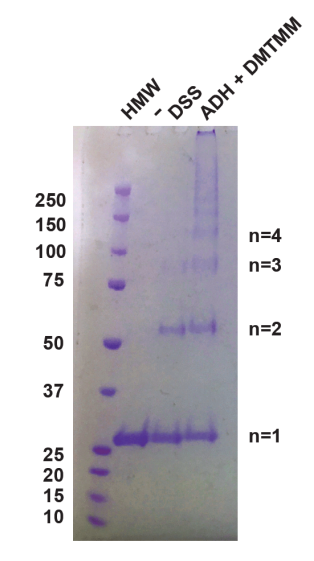

k

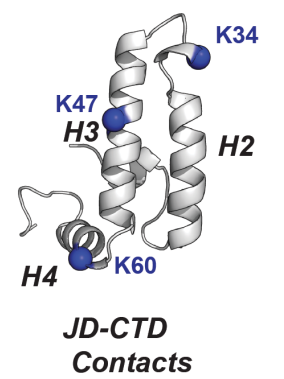

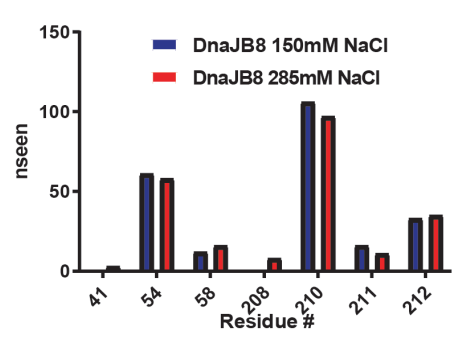

Supplementary Figure 1. Biochemical and mass spectrometry analysis of DnaJB8 in cells and in vitro. (a) Western blot analysis of 1x DnaJB8-Clover, 3x DnaJB8-Clover, 1x Clover and $3 x$ Clover cell lines to quantify levels of expression in each cell line. GAPDH western blot is shown as a loading control. (b) Normalized FITC intensity analysis of 1x Clover (red), 3x Clover (blue), 1x DnaJB8-Clover (purple) and 3x DnaJB8-Clover (green) cell lines. Intensity measurements were calculated from 50 images for each cell line and the signal normalized to DAPI fluorescence intensity. (c) Western blot analysis of input and anti-GFP nanobody elutions DnaJB8-Clover, Clover and HEK293 cell lines as detected with anti-DnaJB8 and anti-GFP antibodies. Total (T) and elutions (E) for each condition are shown. (d) Western blot analysis of DnaJB8-Clover and 
1437 Clover proteins isolated from mammalian cell lines. Total $(T)$, elutions $(E)$ and crosslinked elutions $(\mathrm{XL})$ of each condition are shown. Western blot was probed with GFP antibodies. (e) JD intradomain ADH/DMTMM crosslinks identified from DnaJB8-Clover isolated from cells mapped onto the JD structure. JD is shown in cartoon representation and is colored in white. Sites of crosslink are shown as spheres and are colored red or blue for aspartic/glutamic and lysines, respectively. Dashed yellow lines connect linked amino acid pairs. (f) DnaJB8 particles with a $R_{h}$ of $1-10 \mathrm{~nm}$ in the DLS data were analyzed by proportion of the total sample (\% mass) and binned by the size distribution of the constituent particles. The time evolution of this mass fraction is shown for $150 \mathrm{mM} \mathrm{NaCl}$ (black) and $285 \mathrm{mM} \mathrm{NaCl}$ buffer conditions in 1xPBS. (g) SDS-PAGE coomassie gel showing DnaJB8 in the absence of crosslinker (left), with DSS (middle), and with DMTMM and ADH (right). (h) XL-MS contact map of DnaJB8 crosslinks identified using DMTMM and ADH from a time course: $t=0 \mathrm{hrs}$ (large black dot), $t=10 \mathrm{hrs}$ (medium green dot) and $t=20 \mathrm{hrs}$ (small blue dot), small. The axes are colored in red and green for JD and CTD, respectively. Crosslink pairs between JD-CTD are shown in dashed box colored grey, red and green, respectively. Helix 5 crosslinks are denoted by H5. (i) JD intra-domain ADH/DMTMM in vitro crosslinks mapped onto the JD structure. JD is shown in cartoon representation and is colored in white. Sites of crosslink are shown as spheres and are colored red or blue for aspartic/glutamic and lysines, respectively. Dashed yellow lines connect linked amino acid pairs. (j) Three in vitro JD-CTD inter-domain ADH/DMTMM crosslinks mapped onto the JD structure. DnaJB8 JD is shown in cartoon representation and is colored in white. JD lysine sites that crosslink to CTD are shown as spheres and are colored blue. (k) Histogram of frequency of ADH monolinks observed in normal and elevated ionic strength XLMS experiments. 
a

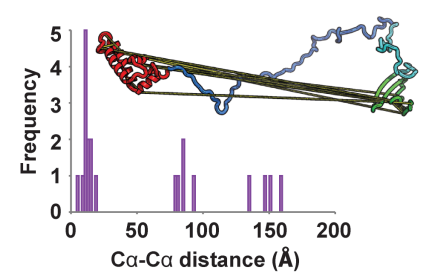

b

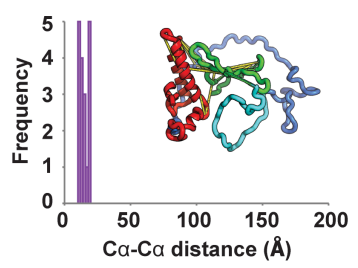

Supplementary Figure 2

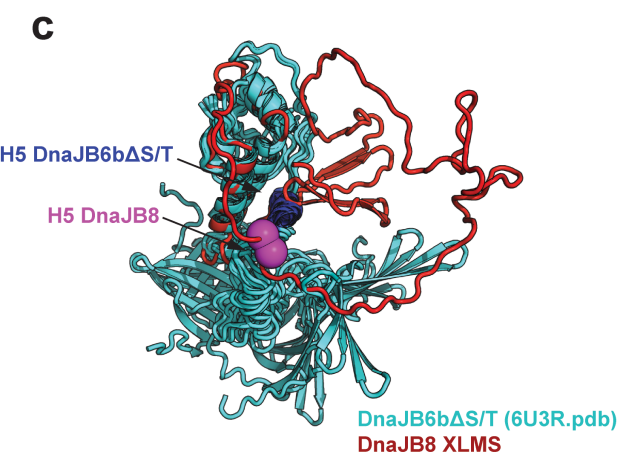

e d

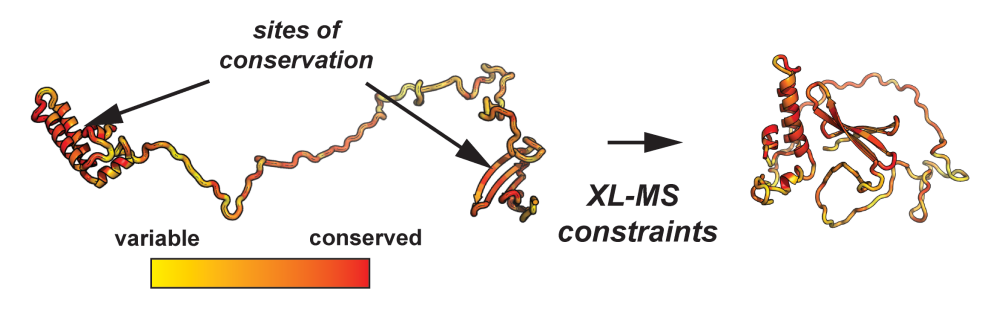

\begin{abstract}
Supplementary Figure 2. Modeling the full length DnaJB8 monomer using crosslinks. (a) DnaJB8 ADH/DMTMM crosslinks mapped onto an expanded structure featuring the conserved JD and our predicted CTD model. The histogram of $C_{\alpha}-C_{\alpha}$ distances shows 11 crosslinks that exceed the physical limitations for ADH/DMTMM. (b) Rosetta ab initio collapsed structure of DnaJB8 with ADH/DMTMM crosslinks mapped. The histogram of $C_{\alpha}-C_{\alpha}$ distances shows that all crosslinks are satisfied when the JD and CTD are docked in this model. (c) Overlay of our Rosetta $a b$ initio collapsed structure (red) with the solution NMR ensemble of the published DnaJB6b $\Delta S T$ deletion variant (cyan; PDBID: 6U3R) The proposed helix $5(\mathrm{H} 5)$ is shown on the DnaJB8 (pink) and DnaJB6b $\Delta$ ST (blue) models. The position of H5 in our DnaJB8 XL-MS-constrained model is close to the position in the DnaJB6b model. The major difference is the position of the CTD, which is influenced by both the XL-MS constraints, and the inclusion of the S/T region that was deleted in the DnaJB6b construct. (d) Conserved surfaces on the JD and CTD in the full-length DnaJB8 model mediate the interaction. Highly conserved sites are colored in red (and highlighted by arrows) and variable positions are colored in yellow. Sequence-based conservation of DnaJB8 shows highly conserved faces along the JD, and some conserved faces in the CTD that overlap with the XL-MS identified surfaces. (e) SDS-PAGE coomassie gel of DnaJB8 and DnaJB8 ${ }_{F \rightarrow S}$ mutant crosslinked with ADH/DMTMM.
\end{abstract}




\section{Supplementary Figure 3}
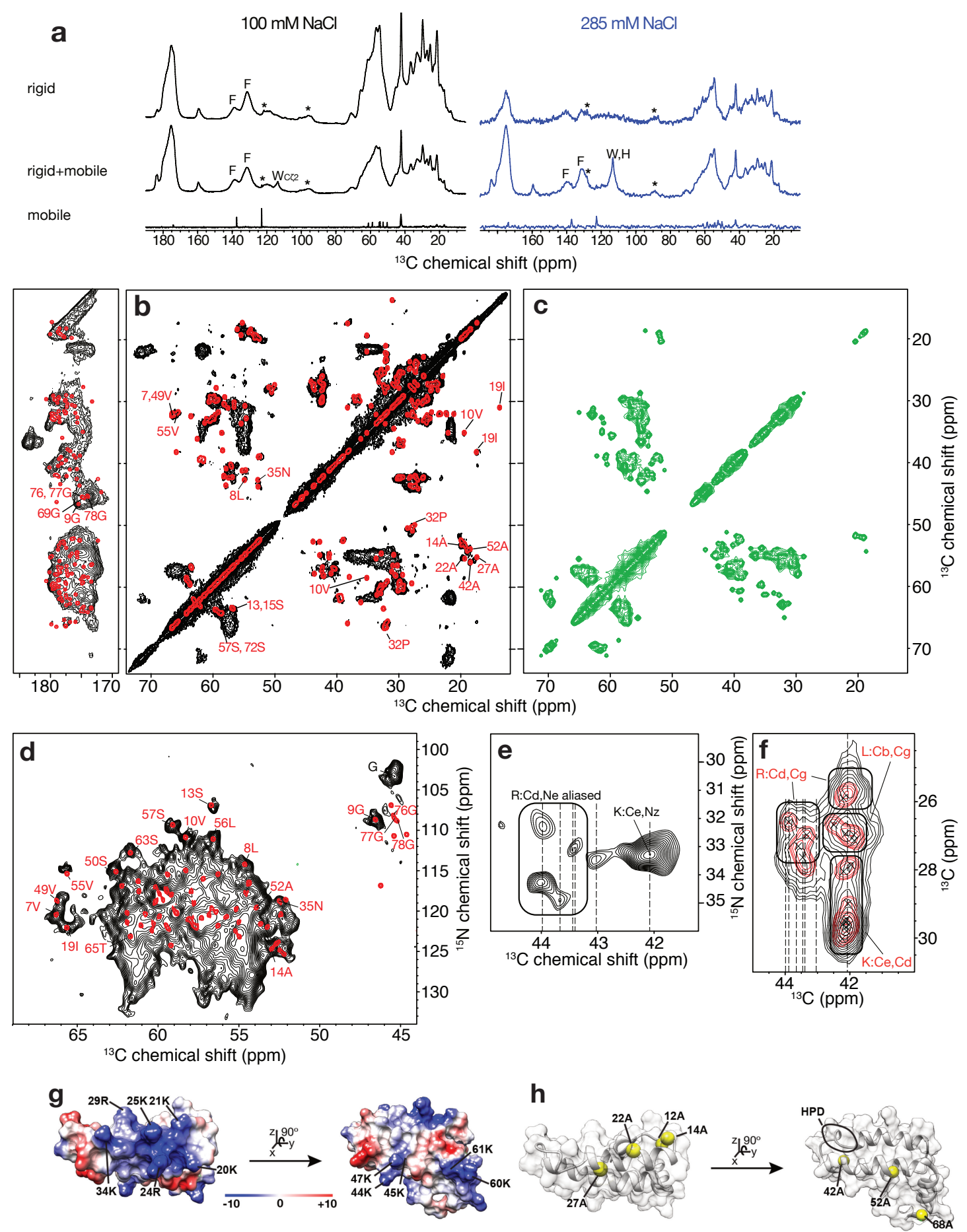
1494 with ${ }^{13} \mathrm{C}$ and ${ }^{15} \mathrm{~N}$ and synthetic (red) spectrum of the DnaJB8 JD in solution generated from BMRB 1495 chemical shifts (red assignments). (c) Simulated spectrum from three other domains (G/F, S/T 1496 and CTD), showing diagonal peaks and one-bond cross-peaks involving backbone $\mathrm{C} \alpha-\mathrm{C} \beta$ and 1497 CO-C $\alpha$ carbons. (d) $2 \mathrm{D}{ }^{13} \mathrm{C}-{ }^{15} \mathrm{~N}$ CP-based NCA experimental spectrum (black) of DnaJB8 1498 oligomers uniformly labeled with ${ }^{13} \mathrm{C}$ and ${ }^{15} \mathrm{~N}$, overlaid with synthetic spectrum of the DnaJB8 JD 1499 in solution (red) from BMRB chemical shifts (red assignments). (e) $2 \mathrm{D}$ CP-based ${ }^{13} \mathrm{C}-{ }^{15} \mathrm{~N}$ spectrum 1500 with $\mathrm{C} \varepsilon-\mathrm{N} \zeta$ and (aliased) $\mathrm{C} \delta-\mathrm{N} \varepsilon$ correlations from immobilized Lysine and Arginine side chains, 1501 respectively. (f) Enlarged ${ }^{13} \mathrm{C}$ - $\mathrm{C}$ spectral region from panel (A) demonstrating good alignment of 1502 Arg, Lys and Leu signals between experimental ssNMR (black) and solution chemical shifts of 1503 the JD (red). Dashed lines mark known ${ }^{13} \mathrm{C}$ positions used to confirm amino acid types of signals. 1504 (g) Electrostatic surface potential of the DnaJB8 JD, with selected residues indicated. Highly 1505 positive and negative potentials are colored blue and red, respectively. White color represents 1506 neutral charge. (h) The same view showing the location of Ala with narrow peaks at $100 \mathrm{mM} \mathrm{NaCl}$ 1507 which disappear at $285 \mathrm{mM} \mathrm{NaCl}$ (Fig. 3, panel "c"). 
a

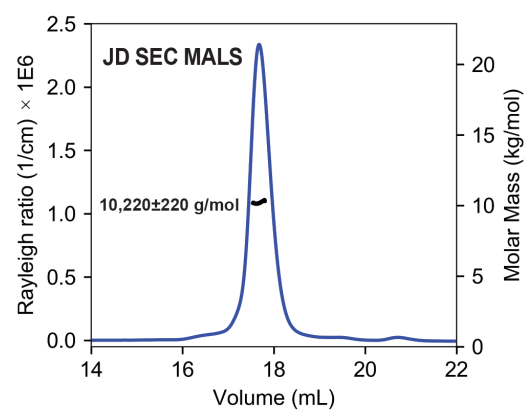

C

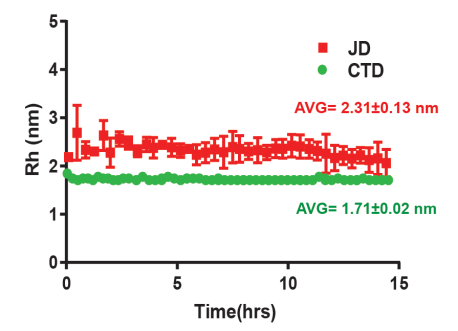

e

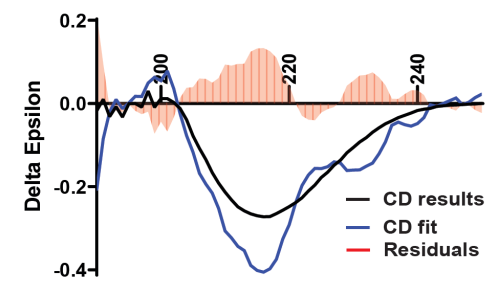

Wavelength $(\mathrm{nm})$

g

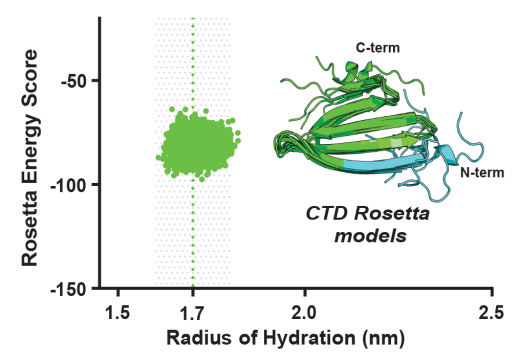

b

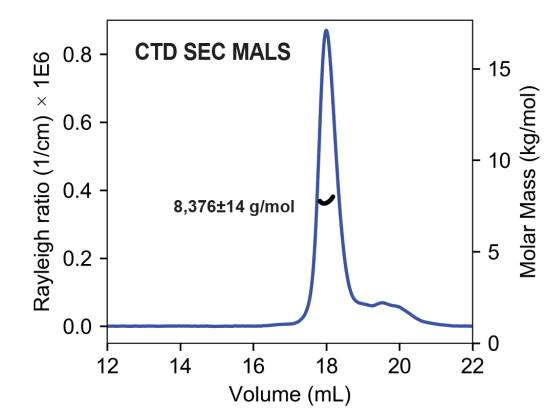

d

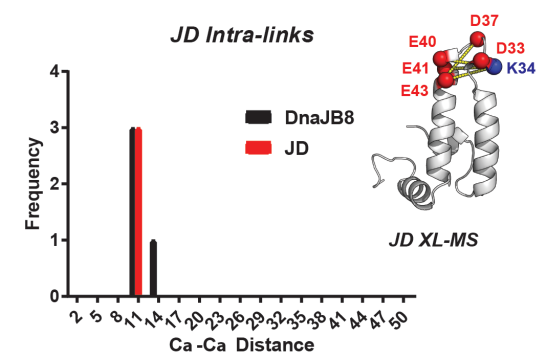

f

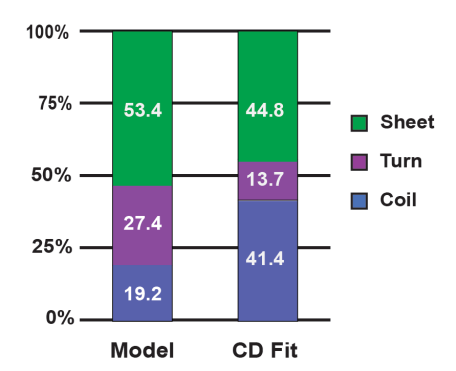

Supplementary Figure 4. JD and CTD are stable monomers. (a) SEC-MALS of JD ${ }_{1-77}$ shows SEC-MALS of CTD ${ }_{170-232}$ shows a single peak with a calculated molar mass of $8,376 \pm 14 \mathrm{~g} / \mathrm{mol}$ consistent with a monomer. (c) DLS time course of $\mathrm{JD}_{1-77}$ and $\mathrm{CTD}_{170-232}$ constructs. The average $\mathrm{R}_{\mathrm{h}}$ of $\mathrm{JD}_{1-77}$ and $C T D_{170-232}$ was calculated to be $2.30 \pm 0.12 \mathrm{~nm}$ and $1.71 \pm 0.02 \mathrm{~nm}$, respectively. (d) Histogram of JD intra-domain crosslinks across XLMS experiments for DnaJB8(black), JD ${ }_{1}$ ${ }_{77}(\mathrm{red})$, and $\mathrm{JD}_{1-77}$ mixed with $\mathrm{CTD}_{170-232}$ (grey). All intra-domain crosslinks satisfy the physical constraint of $<20 \mathrm{~nm}$ for the ADH/DMTMM crosslinkers. (e,f) CD spectra of CTD was analyzed using the Bestsel server. Secondary structure analysis of the data shows high $\beta$-sheet character 
1533 (yellow) with some helix character (purple) and no random coil (blue). The results are compared 1534 to the CTD model generated from ab initio. (g) 5,000 Rosetta ab initio generated models of CTD. 1535 The structural models are consistent with $R_{h}$ values derived from DLS (dashed line). Structural overlay of low energy scoring models reveals consistent pleated beta-sheet fold. Models are 
a

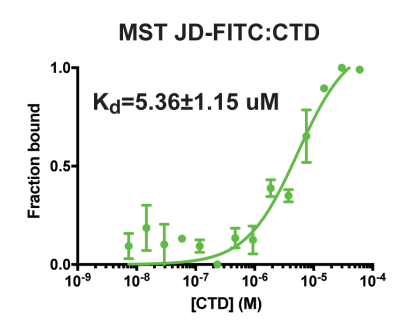

\section{Supplementary Figure 5}

b

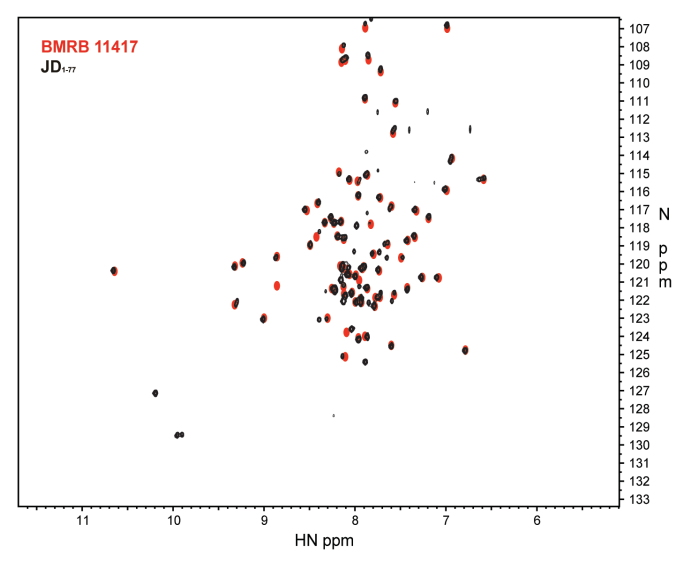

C

Supplementary Figure 5. $\mathrm{JD}_{1-77}$ construct spectra consistent with deposited chemical shifts. (a) Microscale Thermophoresis measurements of JD ${ }_{1-77}-\mathrm{FITC}$ binding to CTD $170-232$. Measurements were performed in triplicate using 200nm JD ${ }_{1-77}-F I T C$ and a range of CTD $170-232$. The samples were incubated and measured in a Monolith NT.115 instrument. The data were processed using PALMIST software and the normalized fluorescence values plotted as an average across three replicates with standard deviations (b) Overlay of our ${ }^{15} \mathrm{~N}-{ }^{1} \mathrm{H} H S Q \mathrm{C}$ spectra

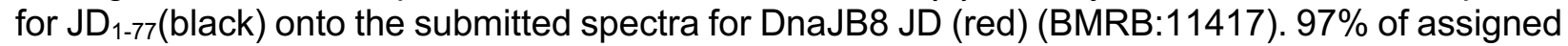
peaks in the published spectra were matched to our data. (c) Amino acid assignments mapped onto the HSQC spectra for the $\mathrm{JD}_{1-77}$ protein.

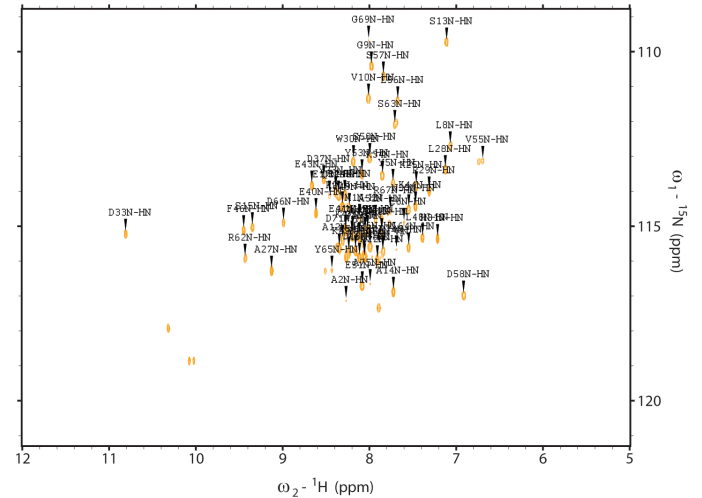

1570

1571 
1572

1573

1574

1575

1576

1577

1578

1579

1580

1581

1582

1583

\section{Supplementary Figure 6}

a

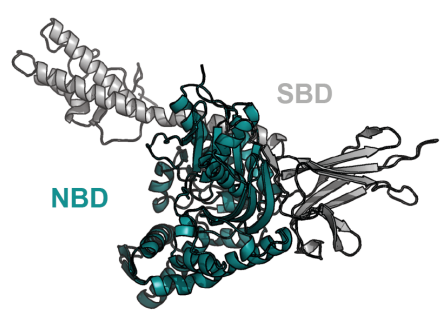

C

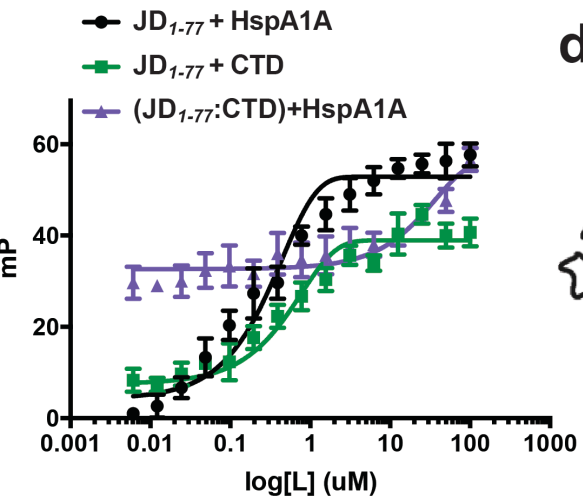

e

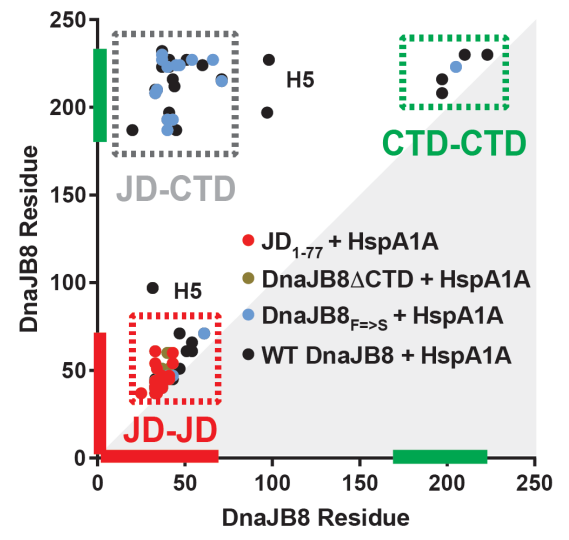

b

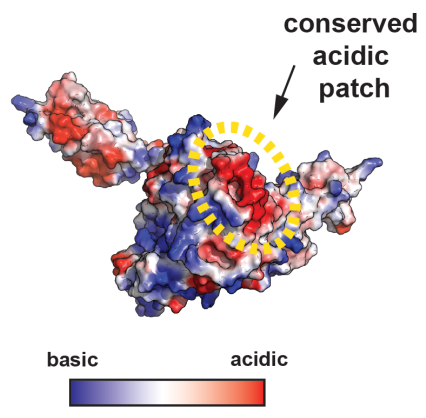

d

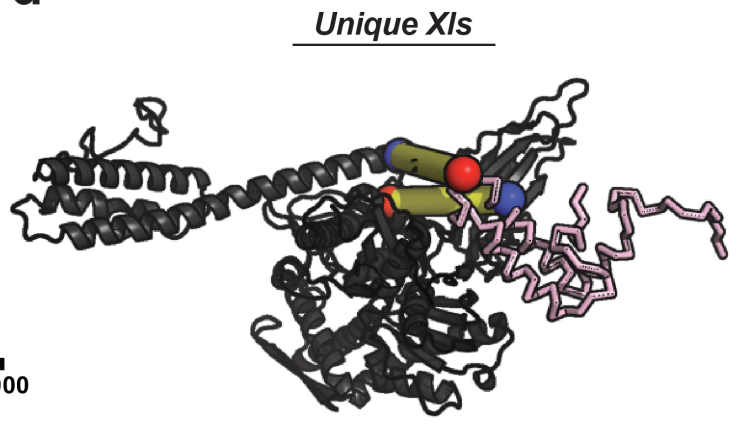

HspA1A:DnaJB8 ${ }_{F \rightarrow S}$

Supplementary Figure 6. Hsp70 proteins present a conserved acidic surface. (a) Structure of HspA1A colored by domain with the nucleotide binding domain (NBD) in turquoise and substrate binding domain (SBD) in grey. (b) Electrostatic surface mapped onto the HspA1A structure with the negatively charged Hsp40-JD-binding site circled in yellow. (c) Raw FP binding curves of labeled JD mixed with HspA1A (black), with CTD (green) and (JD-CTD)+HspA1A (purple). Y-axis is defined as milipolarization (mP). FP experiments were performed in triplicate and shown as averages with standard deviation. (d) The two intermolecular crosslinks identified across three XL-MS datasets between JD and HspA1A in the DnaJB8:HspA1A complex mapped onto JD-HspA1A model. JD is shown in ribbon representation and HspA1A in cartoon representation, colored pink and black respectively. Sites of crosslink are shown as red or blue 
1584 spheres for aspartic/glutamic acid and lysines, respectively. Yellow lines connect linked amino 1585 acid pairs. (e) XL-MS contact map of changes in intramolecular DnaJB8 crosslinks identified using DMTMM and ADH in co-mixtures of different variants of DnaJB8 with HspA1A: WT DnaJB8:HspA1A (black), DnaJB8 $\triangle$ CTD:HspA1A (brown), DnaJB8 ${ }_{F \rightarrow s}: H s p A 1 A$ (blue) and JD ${ }_{1}$ 77:HspA1A (red). The axes are colored in red and green for JD and CTD, respectively. Crosslink pairs between JD-CTD are shown in dashed box colored grey, red and green, respectively. Contacts to helix 5 in WT DnaJB8 are denoted with H5. 